
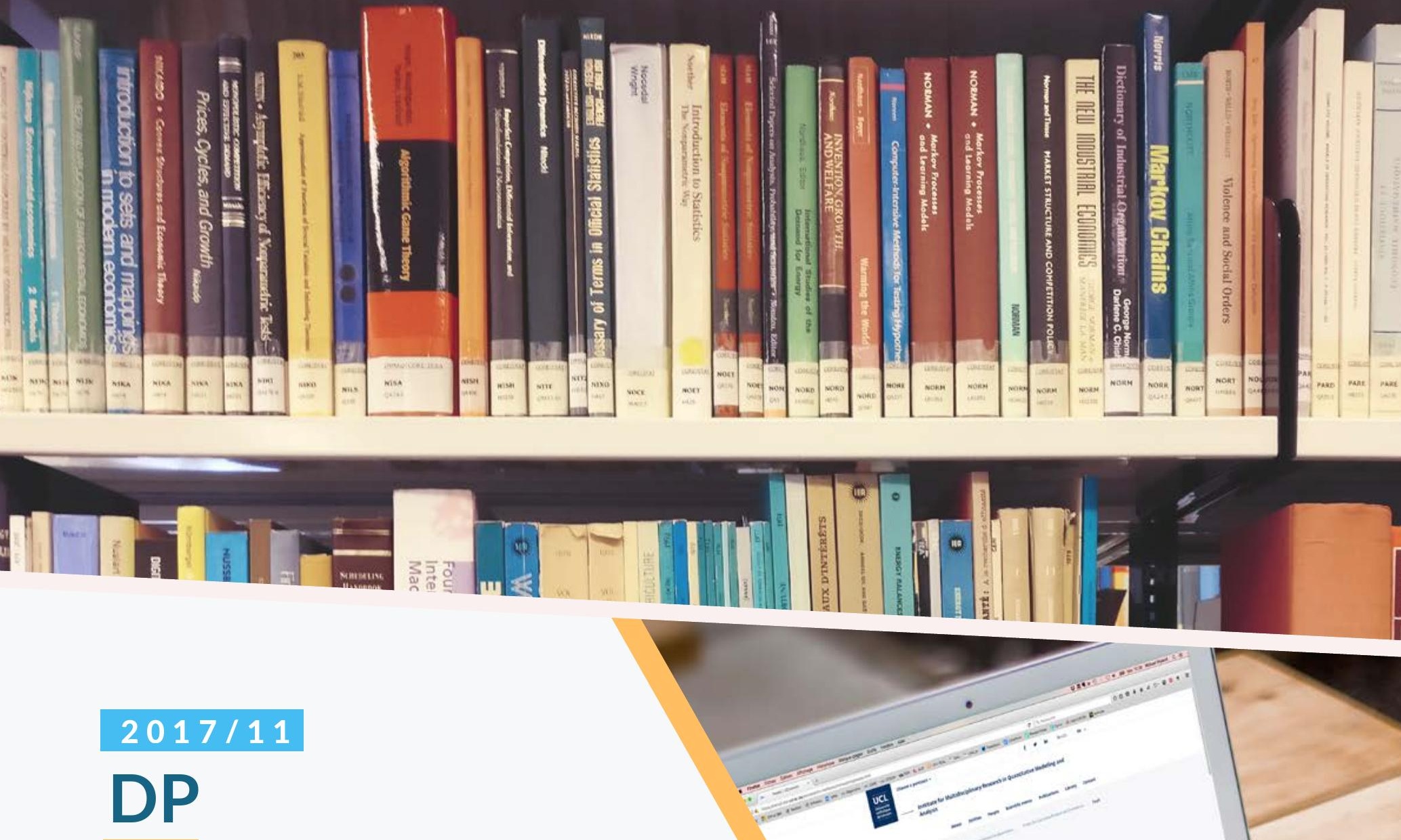

Saptarshi Mukherjee, Nozomu Muto and Eve Ramaekers

Implementation in

undominated strategies with

partially honest agents

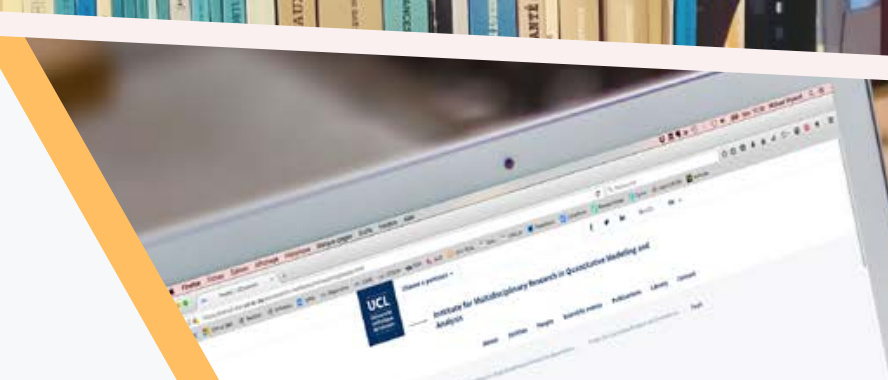




\section{CORE}

Voie du Roman Pays 34, L1.03.01

Tel (32 10) 474304

Fax (32 10) 474301

Email: immaq-library@uclouvain.be

https://uclouvain.be/en/research-institutes/

immaq/core/discussion-papers.html 


\title{
Implementation in Undominated Strategies with Partially Honest Agents*
}

\author{
Saptarshi Mukherjee $^{\dagger} \quad$ Nozomu Muto Eve Ramaekers $^{\S}$
}

March 2, 2017

\begin{abstract}
We consider implementation in undominated strategies by bounded mechanisms. We provide a complete characterization of the class of social choice correspondences that are implementable when agents are partially honest, in the sense that they have strict preferences for being sincere when truthfulness does not result in a worse outcome. As an application, we show that the Pareto correspondence is implemented by a finite mechanism.
\end{abstract}

JEL codes: D82, D71, D01

Keywords: Implementation in undominated strategies, Partial honesty, Bounded mechanism, Pareto correspondence

${ }^{*}$ We thank Salvador Barberá, Claude d'Aspremont, Tadashi Hashimoto, Matt Jackson, Takashi Kunimoto, Jordi Massó, Daisuke Nakajima, Akira Okada, Arunava Sen, Takuma Wakayama, Takahiro Watanabe, Takuro Yamashita, Takehiko Yamato, and seminar/conference participants at Hitotsubashi University, Tokyo Institute of Technology, Summer Workshop in Economic Theory at Sapporo, and the 19th Decentralization Conference in Japan at Yokohama, and anonymous advisory editor and two reviewers for helpful comments. We gratefully acknowledge support from the Spanish Ministry of Science and Innovation through grant "Consolidated Group-C" ECO2008-04756 and FEDER. Muto gratefully acknowledges support from JSPS Grants-in-Aid for Scientific Research No. 26780116.

${ }^{\dagger}$ Department of Humanities and Social Sciences, Indian Institute of Technology-Delhi, Hauz Khas, New Delhi 110016. E-mail: saptarshi.isi@gmail.com

${ }^{\ddagger}$ Department of Economics, Yokohama National University, 79-3 Tokiwadai, Hodogaya-ku, Yokohama 240-8501, Japan. E-mail: nozomu.muto@gmail.com

${ }^{\S}$ Université catholique de Louvain, CORE, Voie du Roman Pays, 34, B-1348 Louvain-la-Neuve, Belgium. E-mail: eve.ramaekers@uclouvain.be 


\section{Introduction}

Implementation theory attempts to structure the interactions amongst the agents by designing a game-form such that in every possible "state of the world", equilibrium actions of agents according to some pre-specified equilibrium notion lead to socially desirable outcomes. In this paper, we consider an incomplete information setting in which each agent has private information about her preference over a finite set of alternatives, and a state of the world is a collection of preferences, one for each agent. In what follows, we focus on the case where her preference is independent of the others' private information. Thus each agent knows her own preference but is ignorant of the preferences of others. The mechanism designer has no information regarding the state of the world.

The literature on implementation theory considers various equilibrium notions. A natural notion of equilibria under the information structure in our setting is dominant strategy equilibrium. The significant advantage of using the dominant strategy concept is that the mechanism designed does not depend on the prior beliefs of the agents. However, dominant strategy implementation is a demanding requirement. For instance, according to the Gibbard-Satterthwaite Theorem (Gibbard (1973), Satterthwaite (1975)), the only social choice functions that can be implemented in a complete domain of preferences are the dictatorial ones (provided that the range of the social choice function has a range of at least three alternatives).

A restriction in the above model is that, at each state of the world, there must be a single socially desirable alternative, as it is defined as a social choice function. In this paper, we relax this assumption, and consider a social choice correspondence (SCC) that allows for multiple socially permissive alternatives at each state of the world. We consider another natural prior-free notion of equilibrium: implementation in undominated strategies by a bounded mechanism. In this notion the agents eliminate (in a single round) their weakly dominated strategies and the union of undominated strategies of all the agents results in the desired social outcomes at each state. The boundedness of the implementing mechanism requires that if a strategy is weakly dominated for at any state, there exists an undominated strategy (at that state) which weakly dominates the former strategy. Thus there exists no sequence of weakly dominated strategies where each strategy weakly dominates the former strategy in the sequence without having any undominated strategy dominating all the strategies. This is an important qualification of the mechanism, and was introduced by Jackson (1992). This influential paper shows that if this requirement is relaxed, then almost every social choice correspondence can be implemented in undominated strategies by a mechanism that uses some integer game structure with tail-chasing construction and hence the mechanism is not 
bounded generally. Jackson (1992) points out several undesirable features of such unbounded mechanisms.

Jackson (1992) investigates the class of SCCs that are implementable in undominated strategies by a bounded mechanism" and provides a necessary condition called "strategy resistance." There are several other papers along this line. Börgers (1991) considers, in the full domain of preferences, implementation of a "compromise," an alternative which is Pareto efficient but does not come at the top of a preference of any agent, in undominated strategies. He shows that it is impossible to implement an SCC which is Pareto efficient and assigns only compromises for some preference profile, if either there are only two agents or there are only three alternatives. Yamashita (2015) provides several applications to auctions and bilateral trade. In some cases one can accomplish more using undominated-strategy than dominant-strategy mechanisms. ${ }^{2}$ While these papers have investigated several interesting facets of implementation in undominated strategies, a complete characterization of the class of implementable SCCs has remained elusive. The natural question remains: what is the class of SCCs implementable in undominated strategies by bounded mechanisms? We aim to answer this question under a particular assumption on behavior of the agents.

In this paper, we investigate the properties of implementable SCCs within a particular framework: when the agents are "partially honest". Informally, a partially honest agent prefers being more "sincere" unless she is strictly better off being less "sincere". In this sense, the agents have an intrinsic preference for honesty or sincerity, which lexicographically ranks below the preference over the alternatives. Although we will introduce a formal notion of sincerity later in Section 2, we give a glimpse of it here. In this paper we consider a class of mechanisms where as a component of a message, an agent has to report her preference ordering many times. We say that a message $m_{i}$ by an agent is more sincere than another message $m_{i}^{\prime}$ if she reports false preferences less times in $m_{i}$ than in $m_{i}^{\prime}$. If $m_{i}$ and $m_{i}^{\prime}$ produce the same outcome against a message profile of the others, say $m_{-i}$, then a partially honest agent strictly prefers $\left(m_{i}, m_{-i}\right)$ to $\left(m_{i}^{\prime}, m_{-i}\right)$. Thus the agents have preference over the outcomes as well as the messages in a mechanism. We will prove our main result for this particular notion of degree of sincerity, but we show in Section 4 that the result holds good for a broader definition of degree of sincerity by identifying a set of sufficient conditions that a sincerity ordering should satisfy for the result to hold good. We also provide an example

\footnotetext{
${ }^{1}$ Henceforth by "implementation" of an SCC we mean implementation in undominated strategies by a bounded mechanism, unless otherwise stated.

${ }^{2}$ Ohseto (1994) considers the plurality correspondence in a voting environment, and proves some impossibility results in specific environments (in term of the number of agents and alternatives). Börgers and Smith (2012) analyse models of bilateral trade and voting, and show that if agents play undominated strategies, a mechanism outperforms any strategy-proof mechanism. Carroll (2014) considers complexity issues in this implementation problems and proves a negative result.
} 
of another degree of sincerity belonging to this class

Honesty of agents has been widely discussed in sociology and social psychology literature. Bok (1999) provides a moral analysis of lying and opines that "truthful statements are preferable to lies in the absence of special considerations." McCornack (1997) argues that lying is generally employed as a strategic option of last resort. Levine et al. (2010) conduct social experiments to test these hypotheses, and establish that people deceive only when doing so yields a benefit. Papers in behavioral and experimental economics establish similar results. Gneezy (2005) conducts an experimental study and shows that a significant fraction of people are averse to lying. Hurkens and Kartik (2009) run similar experiments and show that either a person will never choose to lie, or a person will lie if the outcome obtained by lying is preferred to the outcome obtained by telling the truth.

We provide an example where partial honesty is natural. Suppose that the municipal corporation of a city needs to shortlist a set of locations for building a public park in the city, and the authority can choose from a set $A$ of possible locations. The corporation must take into account of preferences of the citizens of the city. Each citizen has a preference ordering over possible alternatives in $A$ and the preference ordering takes into account of the net valuations (benefit net of her cost share) of all options. In this setting the assumption of partial honesty is quite natural: if an agent does not gain by lying, she is strictly better off picking a truthful message as it would make her feel socially responsible. Consider any citizen $i$ and two messages $m_{i}$ and $m_{i}^{\prime}$ where $m_{i}$ has higher degree of sincerity. Suppose that if the other citizens choose $m_{-i}$, irrespective of $i$ choosing $m_{i}$ or $m_{i}^{\prime}$, the outcome remains the same. Here even if the outcome is not desirable to $i$, she prefers to choose $m_{i}$ as it would reflect her benevolent nature.

In this framework we provide a complete characterization of the class of SCCs that are implementable. In particular, we provide a condition which we call strong chain dominance and show that it is necessary and sufficient for an SCC $F$ to be implementable. To give a rough overview of the condition let us illustrate with an example in the two-agent case. For each agent $i$, an infinite sequence of preferences $C_{i}=\left(P_{i}^{1}, P_{i}^{2}, \ldots\right)$ is called a chain of agent $i$. Strong chain dominance requires that for each alternative $a \in F\left(P_{1}^{1}, P_{2}^{1}\right)$, there is a "selection" from the SCC F. Let the matrix in Figure 1 represent the selection, where for each $r=1,2, \ldots$ and $c=1,2, \ldots, z_{r, c} \in F\left(P_{1}^{r}, P_{2}^{c}\right)$ is selected among the alternatives specified by $F$ at profile $\left(P_{1}^{r}, P_{2}^{c}\right)$. We note that $z_{1,1}=a$, i.e. the selection for the profile $\left(P_{1}^{1}, P_{2}^{1}\right)$ is a itself: this is a part of strong chain dominance. The most important part of strong chain dominance establishes a dominance relation in the following sense: for each $(r, c)$, it must be that $z_{r+1, c}$ is weakly preferred to $z_{r, c}$ with respect to $(r+1)$ th preference of agent 1 , and $z_{r, c+1}$ is weakly preferred to $z_{r, c}$ with respect to $(c+1)$ th preference of agent 


\begin{tabular}{c|cccc} 
& $P_{2}^{1}$ & $P_{2}^{2}$ & $P_{2}^{3}$ & $\cdots$ \\
\hline$P_{1}^{1}$ & $a$ & $z_{12}$ & $z_{13}$ & $\cdots$ \\
$P_{1}^{2}$ & $z_{21}$ & $z_{22}$ & $z_{23}$ & $\cdots$ \\
$P_{1}^{3}$ & $z_{31}$ & $z_{32}$ & $z_{33}$ & $\cdots$ \\
$\vdots$ & $\vdots$ & $\vdots$ & $\vdots$ & $\ddots$
\end{tabular}

Figure 1: The matrix given by the strong chain dominance condition.

2. Thus, any alternative in the matrix must be weakly preferred to the alternative in the same column (row) but in the previous row (column) according to the preference ordering corresponding to the row (column) of that alternative.

The proof of sufficiency is constructive. We give an informal overview of the implementing mechanism here, again focusing on the two-agent case for notational simplicity. The mechanism is constructed by a step-by-step procedure. We start with an initial mechanism in which every agent $i$ calls an integer $\alpha_{i}$ together with a targeted preference $P_{i}$, and the implemented alternative is determined by a modulo game so that the alternative is one of those specified by the SCC (called a "desired alternative") at preference profile $\left(P_{1}, P_{2}\right)$. In this game, some alternatives which do not belong to the set specified by the SCC F (called "undesired alternatives") could also be implemented. To see this, suppose that an alternative $a$ is the one desired at preference profile $P^{1}=\left(P_{1}^{1}, P_{2}^{1}\right)$. Even when $a$ is undesired at another preference profile $P^{2}=\left(P_{1}^{2}, P_{2}^{2}\right)$, a can also be implemented at $P^{2}$ because for each $i$, the messages implementing $a$ at $P_{i}^{1}$ may be undominated also at $P_{i}^{2}$. The next step is to add messages to this initial mechanism so that the messages implementing undesired alternatives are dominated by the added messages. Here, the alternatives implemented by the added messages are given by the second row or column in Figure 1. The conditions of strong chain dominance allow the added messages to maintain the required dominance relationship. In particular, each message $\left(\alpha_{i}, P_{i}^{1}\right)$ which is undominated at $P_{i}^{1}$ in the initial mechanism is dominated at $P_{i}^{2}$ by an added message denoted by $\left(\alpha_{i}, P_{i}^{1}, P_{i}^{2}\right)$. The added messages, again, may still implement undesired alternatives. Then, we add messages to the second mechanism so that the messages implementing undesired alternatives are dominated by the added messages. The alternatives implemented by the added messages are given by the third row or column in Figure 1. In particular, each message $\left(\alpha_{i}, P_{i}^{1}, P_{i}^{2}\right)$ in the second mechanism is dominated at $P_{i}^{3}$ by an added message denoted by $\left(\alpha_{i}, P_{i}^{1}, P_{i}^{2}\right)$. This process of adding messages continues infinitely, and thus our implementing mechanism has a countable message space. To sum up, we construct the mechanism by a procedure in which if something goes wrong then we add messages, and if the new messages cause another problem then we repeat the similar process. Our finding is that if the agents are partially honest in 
our sense, the resulting mechanism is bounded. We discuss more details on this in the main sections.

In an important application, we show that the Pareto correspondence, which gathers all Pareto efficient outcomes at each state, satisfies strong chain dominance and hence is implementable in undominated strategies. In particular, the implementing mechanism in this case is shown to be finite. Implementation of Pareto correspondence is interesting in many settings, e.g. the public goods provision example as described above. In this case, the Pareto correspondence is an implementable natural rule following our result if the agents are partially honest.

Several papers discuss the issue of implementation when the agents are "partially honest". Matsushima (2008b) discusses Nash implementation in an incomplete information setting when all the agents are partially honest and shows that (for three or more individuals) each social choice function (SCF) is implementable in the iterative elimination of strictly dominated strategies, if it is Bayesian implementable by a mechanism which is independent of details of the environment such as the agents' priors. In the other paper Matsushima (2008a) proves a similar result in a complete information setting. In these papers, a small but positive cost of lying is assumed, and existence of a small fine is necessary to ensure implementation. The size of the mechanism is finite but crucially depends of the amount of the fine. In contrast, our model requires no such positive fines. Dutta and Sen (2012) prove that, in a complete information setting, if there are three or more individuals and there is at least one partially honest agent, all social choice correspondences satisfying a weak requirement of no veto power can be Nash implemented. Further they show that if everybody is partially honest, then in a separable environment (e.g. classical exchange economy) any SCF is dominant strategy implementable. ${ }^{3}$

Our paper fits into the strand of literature as discussed above, but stands out mainly on two aspects: (a) To the best of our knowledge this is the first complete characterization of SCCs implementable in undominated strategies by a bounded mechanism, albeit in a specific environment (with partially honest agents). The necessary and sufficient condition allows us to identify the class of rules that are implementable. Thus one might explore implementability of rules in various economic environments such as Pareto correspondence,

\footnotetext{
${ }^{3}$ Lombardi and Yoshihara $(2014,2016)$ provide characterizations of Nash implementation, and Lombardi and Yoshihara (2017) provide a characterization of natural implementation in a complete information setting with at least one partially honest agent. Kartik et al. (2014) show that if the environment is complete and satisfies a condition called separable punishment, any SCF is implementable in two rounds of iterative deletion of strictly dominated strategies. Ortner (2015) discusses some refinements of Nash implementation but requires five or more number of agents. Korpela (2014) considers Bayesian implementation of an SCF. Doghmi and Ziad (2013) consider Nash implementation in a fair division problem with a restricted domain such as single-peaked preferences.
} 
the core in exchange economies, stable matching rules, and many others. (b) This work is also the first attempt to discuss the role of partial honesty in an incomplete information environment that uses a belief-free solution concept.

The remainder of the paper proceeds as follows: In Section 2, we introduce our model and define strong chain dominance. In Section 3, we show our main theorem, and provide a condition that guarantees implementation by a finite mechanism (rather than bounded). We also provide several examples of SCCs which satisfy or fail to satisfy strong chain dominance. In Section 4, we argue that our results still hold under different forms of sincerity. Section 5 concludes.

\section{Model}

\section{Social Choice Environment}

We consider a social environment with incomplete information. The society consists of $n$ $(\geq 2)$ agents in $N=\{1, \ldots, n\}$ facing a joint decision problem to choose an alternative. We denote the finite set of feasible alternatives by $A(|A| \geq 2)$. Each agent $i \in N$ makes a decision according to her private information represented by a preference, namely, a binary relation in $A$ which is complete and transitive. We assume that preferences are always strict, and denote the set of all strict preferences by $\mathcal{P} .{ }^{4}$ We write $a P_{i} b$ meaning that agent $i$ with a preference $P_{i} \in \mathcal{P}$ strictly prefers alternative $a$ to $b$. For $P_{i} \in \mathcal{P}$ and $a, b \in A$, we write $a R_{i} b$ meaning either $a P_{i} b$ or $a=b$. We denote the $k$ th ranked alternative among $A^{\prime} \subseteq A$ for an agent at $P_{i}$ by $r^{k}\left(P_{i}, A^{\prime}\right) \in A^{\prime}$. Note that $r^{1}\left(P_{i}, A^{\prime}\right)$ is the top alternative among those in $A^{\prime}$ for an agent with $P_{i}$. The domain of preferences may be restricted. Let $\mathcal{D}_{i} \subseteq \mathcal{P}$ be the set of all possible preferences of agent $i$, and $\mathcal{D}=\mathcal{D}_{1} \times \cdots \times \mathcal{D}_{n}$.

A triple $(N, A, \mathcal{D})$ is referred to as a social choice environment, which is fixed throughout the paper. A social choice correspondence $(S C C)$ is a function from $\mathcal{D}$ to $2^{A} \backslash\{\emptyset\}$.

\section{Mechanism and Partially Honest Agents}

The agents' joint decision is made through a mechanism $\Gamma=(M, g)$, which consists of $M=M_{1} \times \cdots \times M_{n}$ where each $M_{i}$ is a set of messages of agent $i$, and $g: M \rightarrow A$ is an assignment function. For the sake of expositional simplicity, we focus on mechanisms with either finite or countably infinite message spaces. In the mechanism, agents play a strategicform game in which each agent $i$ simultaneously chooses a message $m_{i} \in M_{i}$, and then an alternative $g\left(m_{1}, \ldots, m_{n}\right) \in A$ is implemented.

\footnotetext{
${ }^{4}$ Our main result holds true even when the agents may have weak preferences.
} 
We consider partially honest agents who prefer reporting own preference truthfully if the implemented outcomes are indifferent. Given a mechanism $(M, g)$, each agent $i \in N$ has a sincerity ordering $S_{i}^{P_{i}} \in M_{i} \times M_{i}$ at each preference $P_{i} \in \mathcal{D}_{i}$, which ranks the messages in terms of degree of sincerity when $i$ 's true preference is $P_{i}$. For each $i \in N$ and $P_{i} \in \mathcal{D}_{i}, S_{i}^{P_{i}}$ is a transitive and asymmetric ordering which we will define shortly. For each $m_{i}, m_{i}^{\prime} \in M_{i}$ such that $m_{i} S_{i}^{P_{i}} m_{i}^{\prime}$, the agent $i$ (with true preference $P_{i}$ ) is more sincere when she chooses $m_{i}$ rather than $m_{i}^{\prime}{ }^{5}$ The indifferent part of $S_{i}^{P_{i}}$ is denoted by $I\left(S_{i}^{P_{i}}\right)$, i.e., for each $m_{i}, m_{i}^{\prime} \in M_{i}$, $m_{i} I\left(S_{i}^{P_{i}}\right) m_{i}^{\prime}$ if and only if neither $m_{i} S_{i}^{P_{i}} m_{i}^{\prime}$ nor $m_{i}^{\prime} S_{i}^{P_{i}} m_{i}$. We assume that this ordering is known to the mechanism designer.

We primarily focus on a mechanism with a message space $M_{i} \subseteq \Sigma_{i} \times \mathcal{D}_{i} \times \mathcal{D}_{i} \times \ldots$ where $\Sigma_{i}$ is a finite or countable set for each $i \in N$, together with a sincerity ordering defined as follows: for each $P_{i} \in \mathcal{D}_{i}$, each $m_{i}=\left(\sigma_{i}, P_{i}^{1}, P_{i}^{2}, \ldots\right) \in M_{i}$ and each $\hat{m}_{i}=\left(\hat{\sigma}_{i}, \hat{P}_{i}^{1}, \hat{P}_{i}^{2}, \ldots\right) \in M_{i}{ }^{6}$

$$
m_{i} S_{i}^{P_{i}} \hat{m}_{i} \text { if and only if }\left|\left\{t_{i} \mid P_{i}^{t_{i}} \neq P_{i}\right\}\right|<\left|\left\{t_{i} \mid \hat{P}_{i}^{t_{i}} \neq P_{i}\right\}\right|
$$

This definition captures the following idea: if an agent is requested to report her preference many times (along with a certain element chosen from $\Sigma_{i}$ ), she will feel herself less sincere as she tells lies more frequently. ${ }^{7}$ For any $\sigma_{i} \in \Sigma_{i}$, a message $\left(\sigma_{i}, P_{i}, P_{i}, P_{i}, \ldots\right)$ is a report with a maximum sincerity at $P_{i}$.

Next, we extend an individual agent's preference over $A$ to a lexicographic ordering $\succsim_{i}^{P_{i}}$ over the set of message profiles $M$ : Let $\succsim_{i}^{P_{i}}$ be an ordering over $M$ at preference $P_{i} \in \mathcal{D}_{i}$. The strict part is denoted by $\succ_{i}^{P_{i}}$, and the indifferent part by $\sim_{i}^{P_{i}}$.

Definition 1. In a mechanism $(M, g)$, an agent $i \in N$ is partially honest if for all $P_{i} \in \mathcal{D}_{i}$, and all $\left(m_{i}, m_{-i}\right),\left(m_{i}^{\prime}, m_{-i}\right) \in M$,

(i) if $g\left(m_{i}, m_{-i}\right) P_{i} g\left(m_{i}^{\prime}, m_{-i}\right)$, then $\left(m_{i}, m_{-i}\right) \succ_{i}^{P_{i}}\left(m_{i}^{\prime}, m_{-i}\right)$,

(ii) if $g\left(m_{i}, m_{-i}\right)=g\left(m_{i}^{\prime}, m_{-i}\right)$ and $m_{i} S_{i}^{P_{i}} m_{i}^{\prime}$, then $\left(m_{i}, m_{-i}\right) \succ_{i}^{P_{i}}\left(m_{i}^{\prime}, m_{-i}\right)$, and

(iii) if $g\left(m_{i}, m_{-i}\right)=g\left(m_{i}^{\prime}, m_{-i}\right)$ and $m_{i} I\left(S_{i}^{P_{i}}\right) m_{i}^{\prime}$, then $\left(m_{i}, m_{-i}\right) \sim_{i}^{P_{i}}\left(m_{i}^{\prime}, m_{-i}\right)$.

${ }^{5}$ This definition is a generalization of the notion of partial honesty introduced by Dutta and Sen (2012). Our definition is equivalent to theirs if for each $i \in N$ and each $P_{i} \in \mathcal{D}_{i}$, there exists $M_{i}^{\prime}, M_{i}^{\prime \prime} \subset M_{i}$ such that $M_{i}^{\prime} \cup M_{i}^{\prime \prime}=M_{i}, M_{i}^{\prime} \cap M_{i}^{\prime \prime}=\emptyset$, and $m_{i}^{\prime} S_{i}^{P_{i}} m_{i}^{\prime \prime}$ if and only if $m_{i}^{\prime} \in M_{i}^{\prime}$ and $m_{i}^{\prime \prime} \in M_{i}^{\prime \prime}$. This is equivalent to saying that the sincerity ordering divides the message space into two; the set of "sincere messages" $M_{i}^{\prime}$ and the set of "insincere messages" $M_{i}^{\prime \prime}$. See Section 4 for a discussion.

${ }^{6}$ For a set $X$ which is either finite or countably infinite, $|X|$ denotes the cardinality of $X$. If $X$ is finite and $\hat{X}$ is countably infinite, we define $|X|<|\hat{X}|$, and if $X$ and $\hat{X}$ are both countably infinite, we define $|X|=|\hat{X}|$.

${ }^{7}$ In Section 3.2, we consider mechanisms in which each agent reports preferences finitely many times, associated with a sincerity ordering which is a restriction of the sincerity ordering $(*)$. In Section 4 , we argue that many other sincerity orderings lead to the same conclusion. 
Throughout the paper, we assume that all agents are partially honest in the sense as defined above. ${ }^{8}$

Dominance relations are defined with respect to this ordering $\succsim_{i}^{P_{i}}$. We say that for $m_{i}, m_{i}^{\prime} \in M_{i}, m_{i}$ (weakly) dominates $m_{i}^{\prime}$ at $P_{i} \in \mathcal{D}_{i}$ if $\left(m_{i}, m_{-i}\right) \succsim_{i}^{P_{i}}\left(m_{i}^{\prime}, m_{-i}\right)$ for all $m_{-i} \in M_{-i}$, and $\left(m_{i}, m_{-i}\right) \succ_{i}^{P_{i}}\left(m_{i}^{\prime}, m_{-i}\right)$ for some $m_{-i} \in M_{-i}$. A message $m_{i}$ is undominated at $P_{i} \in \mathcal{D}_{i}$ if no message dominates $m_{i}$ at $P_{i}$. Let $U_{i}\left(P_{i}, \Gamma\right) \subseteq M_{i}$ be the set of undominated messages of $i$ at $P_{i} \in \mathcal{D}_{i}$ in a mechanism $\Gamma=(M, g)$, and let $U(P, \Gamma)=U_{1}\left(P_{1}, \Gamma\right) \times \cdots \times$ $U_{n}\left(P_{n}, \Gamma\right) \subseteq M$. We sometimes denote $U_{i}\left(P_{i}, \Gamma\right)$ simply by $U_{i}\left(P_{i}\right)$ if the mechanism $\Gamma$ in consideration is evident.

We introduce an important property of a mechanism.

Definition 2. A mechanism $\Gamma=(M, g)$ is bounded if every dominated message is dominated by an undominated message, i.e., for all $i \in N$ and all $P_{i} \in \mathcal{D}_{i}$, if a message $m_{i} \in M_{i}$ is dominated at $P_{i}$ then there exists $m_{i}^{\prime} \in U_{i}\left(P_{i}\right)$ that dominates $m_{i}$ at $P_{i}{ }^{9}$

Remark 1. Dominance relations at preference $P_{i}$ when agent $i$ is not partially honest are defined in a standard manner. To distinguish this from the dominance relations with respect to the orderings given in Definition 1, we refer to the dominance relations when $i$ is not partially honest as dominance relations with respect to material preferences.

Since the ordering given in Definition 1 is a refinement of the ordering with respect to material preferences, for each agent $i \in N$, each preference $P_{i} \in \mathcal{D}_{i}$, and each pair of messages $m_{i}, m_{i}^{\prime} \in M_{i}$ in a mechanism, if $m_{i}^{\prime}$ dominates $m_{i}$ at $P_{i}$ (when $i$ is partially honest), then either (a) $m_{i}^{\prime}$ dominates $m_{i}$ at $P_{i}$ with respect to material preferences, or (b) $m_{i}$ and $m_{i}^{\prime}$ implement the same alternatives for all $m_{-i} \in M_{-i} \cdot{ }^{10}$ Therefore, if $m_{i}$ is undominated at $P_{i}$ with respect to material preferences, but $m_{i}$ is dominated at $P_{i}$, then any message $m_{i}^{\prime}$ dominating $m_{i}$ satisfies (b) above.

Suppose that the mechanism is bounded. Then, there exists a message $\bar{m}_{i}$ which is undominated at $P_{i}$, and maximally sincere at $P_{i}$ in the set of messages satisfying (b). Hence, we have shown that if $m_{i}$ is undominated at $P_{i}$ with respect to material preferences, then

\footnotetext{
${ }^{8}$ We note that this assumption is stronger than the assumption in the main part of Dutta and Sen (2012), in that we assume all (not some) agents are partially honest. We need this assumption because agent $i$ 's undominated strategies are defined according only to $i$ 's preferences.

${ }^{9}$ Boundedness was introduced by Jackson (1992). We note that any finite mechanism is obviously bounded.

${ }^{10}$ We note that (b) relies on the assumption that the preferences are strict. If ties are allowed, there may exist a message profile $m$ and a message $m_{i}^{\prime}$ such that $m$ implements an alternative $a,\left(m_{i}^{\prime}, m_{-i}\right)$ implements another alternative $b \neq a$, and $a$ and $b$ are indifferent at agent $i$ 's preference. If $m_{i}^{\prime}$ is more sincere than $m_{i}$, it is possible that the mechanism implements $a$ with respect to material preferences, but fails to implement $a$ when agents are partially honest. Despite this fact, our main result holds true because in the implementing mechanism we construct, the sincere ordering breaks the above-mentioned indifferences.
} 
there exists $\bar{m}_{i}$ such that $\bar{m}_{i}$ is undominated at $P_{i}$ and $m_{i}$ and $\bar{m}_{i}$ implement the same alternatives for all $m_{-i} \in M_{-i}$.

\section{Implementation in Undominated Strategies}

Given a sincerity ordering, we introduce the notion of implementation in undominated strategies when all agents are partially honest.

Definition 3. A mechanism $\Gamma=(M, g)$ implements a social choice correspondence $F$ in undominated strategies if for each $P \in \mathcal{D}$, we have $F(P)=\{a \in A \mid$ there is $m \in U(P)$ such that $g(m)=a\}$.

We say that an SCC $F$ is implementable in undominated strategies if there exists a bounded mechanism $\Gamma$ that implements $F$ in undominated strategies. The standard notion of implementation in undominated strategies is defined through dominance relations with respect to material preferences. We call this standard notion implementation in undominated strategies with respect to material preferences.

Jackson (1992) introduced a necessary condition of implementability of an SCC F in undominated strategies by a bounded mechanism with respect to material preferences, namely strategy-resistance: An SCC $F$ is strategy-resistant if for each $i \in N$, each $P_{i}, P_{i}^{\prime} \in \mathcal{D}_{i}$, each $P_{-i} \in \mathcal{D}_{-i}$, and each $a \in F\left(P_{i}, P_{-i}\right)$, there exists $b \in F\left(P_{i}^{\prime}, P_{-i}\right)$ such that $b R_{i}^{\prime} a$.

Remark 2. Literature on implementation theory considers two notions of implementation; full implementation in which a set of alternatives is implemented at a state if it coincides with the set of equilibrium outcomes at that state in the mechanism, and partial implementation, in which at any state, the equilibrium outcomes constitute a subset of the outcomes of the SCC at that state. Our Definition 3 adopts full implementation following Jackson (1992), while there are other papers such as Yamashita (2012, 2015) considering partial implementation.

Full implementation is justified from several points of view as discussed by Thomson (1996), while partial implementation is also natural for example when an SCC embodies a certain desirable property, and the social planner is not interested in which alternative is implemented as long as the property is satisfied. If our main theorem is modified properly, we can obtain a necessary and sufficient condition of partial implementation in undominated strategies when all agents are partially honest. ${ }^{11}$

\footnotetext{
${ }^{11}$ Details are upon request.
} 


\section{Strong Chain Dominance}

We introduce a condition called strong chain dominance, which is a strengthened version of "the chain dominance property" introduced by Yamashita (2012). ${ }^{12}$

Let $\mathbb{N}=\{1,2, \ldots\}$ be the set of natural numbers. An infinite sequence $C_{i}=\left(P_{i}^{t_{i}}\right)_{t_{i} \in \mathbb{N}}$ of agent $i$ 's preferences in $\mathcal{D}_{i}$ is called a chain of agent $i$. We denote $C_{i}\left(t_{i}\right)=P_{i}^{t_{i}}$ for each $t_{i} \in \mathbb{N}$. Let $\mathcal{C}_{i}$ be the set of all chains of agent $i$, and let $\mathcal{C}=\prod_{i \in N} \mathcal{C}_{i}$ be the set of all chain profiles.

Definition 4. An SCC $F$ satisfies strong chain dominance if there exists a function $z$ : $A \times \mathbb{N}^{n} \times \mathcal{C} \rightarrow A$ satisfying the following four conditions:

(i) For each $C \in \mathcal{C}$, each $a \in F\left(C_{1}(1), \ldots, C_{n}(1)\right)$, and each $t=\left(t_{1}, \ldots, t_{n}\right) \in \mathbb{N}^{n}$, we have $z(a, t, C) \in F\left(C_{1}\left(t_{1}\right), \ldots, C_{n}\left(t_{n}\right)\right)$.

(ii) For each $C \in \mathcal{C}$ and each $a \in F\left(C_{1}(1), \ldots, C_{n}(1)\right)$, we have $z(a,(1, \ldots, 1), C)=a$.

(iii) For each $C, C^{\prime} \in \mathcal{C}$ and each $t \in \mathbb{N}^{n}$, if $C_{i}\left(\tau_{i}\right)=C_{i}^{\prime}\left(\tau_{i}\right)$ for each $i \in N$ and each $\tau_{i} \leq t_{i}$, then $z(a, t, C)=z\left(a, t, C^{\prime}\right)$ for each $a \in F\left(C_{1}(1), \ldots, C_{n}(1)\right)$.

(iv) For each $C \in \mathcal{C}$, each $a \in F\left(C_{1}(1), \ldots, C_{n}(1)\right)$, each $i \in N$, each $t_{i} \in \mathbb{N} \backslash\{1\}$, and each $t_{-i} \in \mathbb{N}^{n-1}$, we have $z\left(a,\left(t_{i}, t_{-i}\right), C\right) R_{i} z\left(a,\left(t_{i}-1, t_{-i}\right), C\right)$ where $R_{i}$ is the weak order corresponding to $C_{i}\left(t_{i}\right)$.

The first condition specifies the relation of the function $z$ to the SCC $F$. For each chain profile $C \in \mathcal{C}$ and $t \in \mathbb{N}^{n},\left(C_{1}\left(t_{1}\right), \ldots, C_{n}\left(t_{n}\right)\right)$ determines a preference profile. For any $a \in A, z(a, t, C)$ is one of the alternatives specified by $F$ at that preference profile. The second condition says that the selection starts at the given alternative $a$, and the third condition says that the value of $z$ is independent of the preferences that appear after $t_{i}$ for each $i$. The fourth condition requires that for each $i \in N$, we must have the dominance along agent $i$ 's chain with respect to material preferences. This function $z$ will determine the assignment in an implementing mechanism that we will construct in the proof of the main theorem.

To get a feeling of the condition, let us illustrate this function $z$ when $n=2$. Pick a pair of chains $C_{1}=\left(P_{1}^{1}, P_{1}^{2}, \ldots\right) \in \mathcal{C}_{1}$ and $C_{2}=\left(P_{2}^{1}, P_{2}^{2}, \ldots\right) \in \mathcal{C}_{2}$, and an alternative $a \in F\left(P_{1}^{1}, P_{2}^{1}\right)$. Let $z_{t_{1}, t_{2}}=z\left(a,\left(t_{1}, t_{2}\right),\left(C_{1}, C_{2}\right)\right) \in A$. Then, $Z=\left(z_{t_{1}, t_{2}}\right)_{\left(t_{1}, t_{2}\right) \in \mathbb{N}^{2}}$ is an infinite-sized matrix of alternatives. A part of this matrix is shown in Figure 1, which is redisplayed as Figure 2.

\footnotetext{
${ }^{12}$ Yamashita (2015) also discusses the idea of the chain dominance property, although he does not state it explicitly.
} 


\begin{tabular}{c|cccc} 
& $P_{2}^{1}$ & $P_{2}^{2}$ & $P_{2}^{3}$ & $\cdots$ \\
\hline$P_{1}^{1}$ & $a$ & $z_{12}$ & $z_{13}$ & $\cdots$ \\
$P_{1}^{2}$ & $z_{21}$ & $z_{22}$ & $z_{23}$ & $\cdots$ \\
$P_{1}^{3}$ & $z_{31}$ & $z_{32}$ & $z_{33}$ & $\cdots$ \\
$\vdots$ & $\vdots$ & $\vdots$ & $\vdots$ & $\ddots$
\end{tabular}

Figure 2: The matrix $Z$.

Part (i) of strong chain dominance requires that there is a selection from $F$, as represented by the matrix in Figure 2. For each $\left(t_{1}, t_{2}\right) \in \mathbb{N}^{2}, z_{t_{1}, t_{2}}$ is one of the desired alternatives selected at preference profile $\left(P_{1}^{t_{1}}, P_{2}^{t_{2}}\right)$. Part (ii) requires that for each $a \in F\left(P_{1}^{1}, P_{2}^{1}\right)$, such a selection from $F$ exists. (If $a \notin F\left(P_{1}^{1}, P_{2}^{1}\right)$, conditions require nothing.) We note that only a part of the entire selection has been depicted in Figure 2. Part (iii) requires that the part of the selection shown in Figure 2 is independent of the preference orderings appearing after $P_{1}^{3}$ and $P_{2}^{3}$ in the sequences $\left(P_{1}^{1}, P_{1}^{2}, \ldots\right)$ and $\left(P_{2}^{1}, P_{2}^{2}, \ldots\right)$. Part (iv) of the condition establishes a dominance relation: for each $\left(t_{1}, t_{2}\right)$, it must be that $z_{t_{1}+1, t_{2}} R_{1}^{t_{1}+1} z_{t_{1}, t_{2}}$ and $z_{t_{1}, t_{2}+1} R_{2}^{t_{2}+1} z_{t_{1}, t_{2}}$. Thus, any alternative in the matrix must be weakly preferred to the alternative in the same column (row) but in the previous row (column) according to the preference corresponding to the row (column) of that alternative. The above dominance between two alternatives that appear at consecutive cells in the matrix shows that any row (or, column) dominates the previous one with respect to material preferences according to the preference corresponding to the row (column), or these two rows (columns) exhibit the same sequence of alternatives.

Based on this observation, we explain roughly why every implementable SCC satisfies strong chain dominance. Let $(M, g)$ be the bounded mechanism implementing the SCC $F$. Suppose that a chain profile $\left(C_{1}, C_{2}\right)$ is given as above, and $a$ is implemented at $\left(P_{1}^{1}, P_{2}^{1}\right)$. Then, there exists a message profile $\left(m_{1}^{1}, m_{2}^{1}\right)$ implementing $a$. For each $i$ and each $t_{i} \in \mathbb{N}, m_{i}^{t_{i}}$ is either dominated or undominated at $P_{i}^{t_{i}+1}$. If dominated, let $m_{i}^{t_{i}+1}$ be the message which dominates $m_{i}^{t_{i}}$ and is undominated at $P_{i}^{t_{i}+1}$. (Boundedness guarantees existence of such a message.) Otherwise, let $m_{i}^{t_{i}+1}=m_{i}^{t_{i}}$. This recursively defines a sequence of messages for each $i$, and induces a matrix $Z=\left(z_{t_{1}, t_{2}}\right)_{\left(t_{1}, t_{2}\right) \in \mathbb{N}^{2}}$ given by $z_{t_{1}, t_{2}}=g\left(m_{1}^{t_{1}}, m_{2}^{t_{2}}\right)$. Since $m_{i}^{t_{i}}$ is undominated at $P_{i}^{t_{i}}$, condition (i) of strong chain dominance holds. Since $\left(m_{1}^{1}, m_{2}^{1}\right)$ implements $a$, condition (ii) holds. Since the definition of $m_{i}^{t_{i}}$ is independent of $m_{i}^{t_{i}+1}$ or later, condition (iii) holds. By the dominance relation between $m_{i}^{t_{i}+1}$ and $m_{i}^{t_{i}}$, condition (iv) holds.

We now explain roughly how strong chain dominance is related to construction of implementing mechanism. By employing a modulo structure, we can easily construct an "initial" 
mechanism that implements all desired alternatives, but may also implement undesired alternatives. For each agent $i \in N$, a typical message in this initial mechanism is denoted as $\left(\alpha_{i}, P_{i}\right)$ where $\alpha_{i}$ is an integer used in the modulo structure, and $P_{i}$ is the targeted preference at which the message wants to implement a desired alternative. Pick two chains $C_{1}=\left(P_{1}^{1}, P_{1}^{2}, \ldots\right) \in \mathcal{C}_{1}$ and $C_{2}=\left(P_{2}^{1}, P_{2}^{2}, \ldots\right) \in \mathcal{C}_{2}$ such that for each $i \in N$ and $t_{i} \in \mathbb{N}$, $P_{i}^{t_{i}} \neq P_{i}^{t_{i}+1}$. Let $a \in F\left(P_{1}^{1}, P_{2}^{1}\right)$ be a desired alternative at $\left(P_{1}^{1}, P_{2}^{1}\right)$. Suppose that a message profile $\left(\left(\alpha_{1}, P_{1}^{1}\right),\left(\alpha_{2}, P_{2}^{1}\right)\right)$ in the initial mechanism implements $a$, and for each $i$, a message $\left(\alpha_{i}, P_{i}^{1}\right)$ in the initial mechanism is undominated at $P_{i}^{1}$. It is possible that for each $i,\left(\alpha_{i}, P_{i}^{1}\right)$ is undominated also at $P_{i}^{2} \neq P_{i}^{1}$, where $a \notin F\left(P_{1}^{2}, P_{2}^{2}\right)$. In such a case, the initial mechanism implements an undesired alternative at $\left(P_{1}^{2}, P_{2}^{2}\right)$. To solve this problem, for each $i$, we add a message $\left(\alpha_{i}, P_{i}^{1}, P_{i}^{2}\right)$ which implements the second row or column in the matrix in Figure 2. Since $\left(\alpha_{i}, P_{i}^{1}\right)$ and $\left(\alpha_{i}, P_{i}^{1}, P_{i}^{2}\right)$ lie once at $P_{i}^{2}$, these messages are equally sincere at $P_{i}^{2}$. By condition (iv) of strong chain dominance, the added message $\left(\alpha_{i}, P_{i}^{1}, P_{i}^{2}\right)$ dominates $\left(\alpha_{i}, P_{i}^{1}\right)$ at $P_{i}^{2}$. This dominance solves the problem that $\left(\left(\alpha_{1}, P_{1}^{1}\right),\left(\alpha_{2}, P_{2}^{1}\right)\right)$ implements $a \notin F\left(P_{1}^{2}, P_{2}^{2}\right)$ at $\left(P_{1}^{2}, P_{2}^{2}\right)$.

The added message $\left(\alpha_{i}, P_{i}^{1}, P_{i}^{2}\right)$, however, may be undominated at another preference $P_{i}^{3} \neq P_{i}^{2}$. If $z_{22} \notin F\left(P_{1}^{3}, P_{2}^{3}\right)$, this causes a new problem that $\left(\left(\alpha_{1}, P_{1}^{1}, P_{1}^{2}\right),\left(\alpha_{2}, P_{2}^{1}, P_{2}^{2}\right)\right)$ implements $z_{22}$ at $\left(P_{1}^{3}, P_{2}^{3}\right)$. To solve this problem, we add a new message $\left(\alpha_{i}, P_{i}^{1}, P_{i}^{2}, P_{i}^{3}\right)$ which implements the third row or column in the matrix shown in Figure 2. Once again, by condition (iv) of strong chain dominance, the added message dominates $\left(\alpha_{i}, P_{i}^{1}, P_{i}^{2}\right)$ at $P_{i}^{3}$, and this dominance solves the problem that $\left(\left(\alpha_{1}, P_{1}^{1}, P_{1}^{2}\right),\left(\alpha_{2}, P_{2}^{1}, P_{2}^{2}\right)\right)$ implements $z_{22}$ at $\left(P_{1}^{3}, P_{2}^{3}\right)$. Then, $\left(\alpha_{i}, P_{i}^{1}, P_{i}^{2}, P_{i}^{3}\right)$ may be undominated at $P_{i}^{4}$, and we add $\left(\alpha_{i}, P_{i}^{1}, P_{i}^{2}, P_{i}^{3}, P_{i}^{4}\right)$ which implements the fourth row or column. This procedure of adding messages continues infinitely. Since the resulting message space is countably infinite, it might not be bounded. Our key finding is that this countable mechanism is bounded when the agents are partially honest. We discuss why boundedness follows from partial honesty in Section 3.1.

Before going to the main theorem, we introduce a useful redefinition of the messages discussed above. For each chain $C_{i}=\left(P_{i}^{1}, P_{i}^{2}, \ldots\right)$, and each message in the initial mechanism $\left(\alpha_{i}, P_{i}^{1}\right)$, adding all the messages as above is equivalent to adding a class of messages in which a natural number $t_{i}$ is called together with $\alpha_{i}$ and $C_{i}$. This is because for each $t_{i}$, it is possible to identify the triplet $\left(\alpha_{i}, t_{i}, C_{i}\right)$ as the message $\left(\alpha_{i}, P_{i}^{1}, P_{i}^{2}, \ldots, P_{i}^{t_{i}}\right)$ in the mechanism constructed in the previous paragraph. Note that this identification relies on condition (iii) of strong chain dominance. Thus, we construct the implementing mechanism with messages typically written as $\left(\alpha_{i}, t_{i}, C_{i}\right)$, which is a more convenient way of expressing messages. In the next section, we will make a more formal discussion on how to construct the implementing mechanism and how partial honesty works in that mechanism. 
Remark 3. If conditions (i)-(iv) in Definition 4 are satisfied only in a subset of $\mathcal{C}$, one can obtain a property that is weaker than strong chain dominance. The chain dominance property defined by Yamashita (2012) considers finite sequences that do not contain the same preference twice or more (thus the length of the sequence is at most $\left|\mathcal{D}_{i}\right|$ ). This is equivalent to focusing on chains $C_{i}$ such that $C_{i}\left(t_{i}\right) \neq C_{i}\left(t_{i}^{\prime}\right)$ whenever $t_{i}<t_{i}^{\prime} \leq\left|\mathcal{D}_{i}\right|$, and $C_{i}\left(t_{i}\right)=C_{i}\left(\left|\mathcal{D}_{i}\right|\right)$ for all $t_{i} \geq\left|\mathcal{D}_{i}\right|$. In our definition, the chains are infinitely long, and can contain the same preference twice or more. In such a case, we allow for $z(a, t, C) \neq z\left(a, t^{\prime}, C\right)$ even when $C(t)=C\left(t^{\prime}\right)$. This distinction makes our condition stronger than the chain dominance property.

As Yamashita (2012) points out, strategy-resistance is a special case of chain dominance (and also strong chain dominance) in a restricted set of chain profiles $C \in \mathcal{C}$ satisfying the following condition: there exists $i \in N$ such that for each $j \in N$ and $t_{j}, t_{j}^{\prime} \in \mathbb{N}$ with $t_{j}<t_{j}^{\prime}$, $C_{j}\left(t_{j}\right) \neq C_{j}\left(t_{j}^{\prime}\right)$ if and only if $j=i$ and $t_{j}=1$.

Let us introduce another condition in the following definition:

Definition 5. An SCC $F$ satisfies top-inclusiveness if $F\left(P_{i}, P_{-i}\right)$ contains the top alternative $r^{1}\left(P_{i}, A\right)$ of agent $i$ for all $i \in N$, all $P_{i} \in \mathcal{D}_{i}$, and all $P_{-i} \in \mathcal{D}_{-i} \cdot{ }^{13}$

By taking $z(a, t, C)=a$ if $t=(1, \ldots, 1)$, and $z(a, t, C)=r^{1}\left(C_{j}\left(t_{j}\right), A\right)$ otherwise, where $j=\min \left\{i \in N \mid t_{i}>1\right\}$, one can see that conditions (i)-(iv) in Definition 4 are satisfied, hence top-inclusiveness implies strong chain dominance. The converse is not true. Thus, top-inclusiveness is stronger than strong chain dominance.

\section{$3 \quad$ Results}

\subsection{Main Theorem}

Theorem 1. Suppose that all agents are partially honest with sincerity ordering $(*)$. Then, strong chain dominance is a necessary and sufficient condition of implementability in undominated strategies by a bounded mechanism.

We postpone the proof to Appendix A.1. We discuss construction of the implementing mechanism when strong chain dominance is satisfied. The implementing mechanism has a message space $M_{i}$ contained in $\mathbb{N} \times \mathbb{N} \times \mathcal{C}_{i}$ for each $i \in N$, where a message of agent $i$ is typically written as $\left(\alpha_{i}, t_{i}, C_{i}\right)$. For each $(\alpha, t, C)=\left(\alpha_{i}, t_{i}, C_{i}\right)_{i \in N} \in M=\prod_{i \in N} M_{i}$, the

\footnotetext{
${ }^{13}$ Koray and Slinko (2008) argue that such an SCC maintains flexibility of social choices to rule out a situation where some agent's best alternative is constitutionally deprived.
} 
assigned alternative is given by $z\left(a_{\alpha}, t, C\right)$ where $z$ is the function in Definition 4 , and $a_{\alpha}$ is an alternative determined through a certain modulo structure.

The implementing mechanism is described in the following steps: We start with an initial mechanism in which each message of agent $i$ has a form of $\left(\alpha_{i}, 1,\left(P_{i}, P_{i}, \ldots\right)\right) \in$ $M_{i}$ where $\left(P_{i}, P_{i}, \ldots\right) \in \mathcal{C}_{i}$ is a constant chain. For each message profile $\left(\left(\alpha_{1}, \ldots, \alpha_{n}\right),(1, \ldots, 1),(P, P, \ldots)\right) \in M$, the integers $\alpha_{1}, \ldots, \alpha_{n}$ choose an alternative $a_{\alpha}$ through a modulo structure. Conditions (i) and (ii) in Definition 4 guarantee $a_{\alpha} \in F(P)$. By the modulo structure, $a_{\alpha}$ covers entire $F(P)$ as $\alpha=\left(\alpha_{1}, \ldots, \alpha_{n}\right)$ changes. Thus, all the alternatives assigned by the SCC at $P \in \mathcal{D}$ are implemented in undominated strategies. ${ }^{14}$ On the other hand, the initial mechanism may implement the undesired alternatives also, because for some $P^{\prime} \in \mathcal{D},\left(\alpha_{i}, 1,\left(P_{i}, P_{i}, \ldots\right)\right) \in M_{i}$ can be undominated at $P_{i}^{\prime}$ for each $i$, and $(\alpha,(1, \ldots, 1),(P, P, \ldots)) \in M$ implements an undesired alternative at $P^{\prime}$. We note that this argument applies only when $P^{\prime} \neq P$. The next step is to add messages to this initial mechanism so that the messages implementing undesired alternatives are dominated by the added messages. For each $P_{i}^{\prime} \in \mathcal{D}_{i}$, each message $\left(\alpha_{i}, 1,\left(P_{i}, P_{i}, P_{i}, \ldots\right)\right)$ in the initial mechanism is dominated by an added message $\left(\alpha_{i}, 2,\left(P_{i}, P_{i}^{\prime}, P_{i}^{\prime}, P_{i}^{\prime}, \ldots\right)\right)$ at $P_{i}^{\prime}$ because the latter message is more sincere at $P_{i}^{\prime}$ by the definition of $(*)$. Conditions (iii) and (iv) in Definition 4 guarantee this dominance relation. The added messages, again, may still implement undesired alternatives at some $P^{\prime \prime} \neq P^{\prime}$, because $\left(\alpha_{i}, 2,\left(P_{i}, P_{i}^{\prime}, P_{i}^{\prime}, P_{i}^{\prime}, \ldots\right)\right)$ may be undominated at $P_{i}^{\prime \prime}$. We note again that this argument applies only when $P^{\prime \prime} \neq P^{\prime}$ and $P^{\prime} \neq P$. For each $P_{i}^{\prime \prime} \in \mathcal{D}_{i}$, each message $\left(\alpha_{i}, 2,\left(P_{i}, P_{i}^{\prime}, P_{i}^{\prime}, P_{i}^{\prime} \ldots\right)\right)$ is dominated by an added message $\left(\alpha_{i}, 3,\left(P_{i}, P_{i}^{\prime}, P_{i}^{\prime \prime}, P_{i}^{\prime \prime}, P_{i}^{\prime \prime}, \ldots\right)\right)$ at $P_{i}^{\prime \prime}$. This process of adding messages continues infinitely, and thus our implementing mechanism has a countable message space.

Without partial honesty, this method would involve two kinds of difficulties: First, it is possible that an added message dominates messages in the initial mechanism implementing desired alternatives. Partial honesty removes this difficulty because in our construction, the messages $\left(\alpha_{i}, 1,\left(P_{i}, \ldots, P_{i}\right)\right)$ in the initial mechanism are maximally sincere at $P_{i}$, so the added messages cannot dominate the initial messages at $P_{i}$. Second, it could be that the resulting mechanism is unbounded. Partial honesty guarantees boundedness of the mechanism. To see this, note that, by the reasoning in the previous paragraph, we can restrict the message space to those with chains in which if a preference appears at some two consecutive coordinates, that preference continues infinitely onward. If a message has such a property, and the agent lies $L$ times, then all the lies come before $2 L$-th coordinate. Thus, for each

\footnotetext{
${ }^{14}$ We note that our purpose of adopting modulo structure is quite different from those in the literature on (pure-strategy) Nash implementation. We utilize the modulo structure in order to implement the desired alternatives, while the modulo games in Nash implementation rule out undesired alternatives from the set of implemented outcomes.
} 
added message, there exist only finite messages which are more or equally sincere, so that the number of messages dominating the added message must be finite. (See Lemma 10 in Section 4.2 for a related discussion.) This property shows boundedness of the implementing mechanism. In Section 3.2, we present an example in which a simpler exposition of the implementing mechanism is provided albeit in a special case.

\subsection{Finite Mechanisms}

Since a finite mechanism is bounded but not vice versa, one might wonder under what condition an SCC satisfying strong chain dominance is implemented in undominated strategies by a finite mechanism. In this section, we consider finite mechanisms in which for each agent $i \in N$, there exist a finite set $\Sigma_{i}$ and an integer $K_{i} \geq 1$ such that $i$ 's message space is contained in $\Sigma_{i} \times\left(\mathcal{D}_{i}\right)^{K_{i}}$. A sincerity ordering parallel to $\left(^{*}\right)$ is defined by, for each $P_{i} \in \mathcal{D}_{i}$, each $m_{i}=\left(\sigma_{i}, P_{i}^{1}, \ldots, P_{i}^{K_{i}}\right) \in M_{i}$ and each $\hat{m}_{i}=\left(\hat{\sigma}_{i}, \hat{P}_{i}^{1}, \ldots, \hat{P}_{i}^{K_{i}}\right) \in M_{i}$,

$$
m_{i} S_{i}^{P_{i}} \hat{m}_{i} \text { if and only if }\left|\left\{t_{i} \mid P_{i}^{t_{i}} \neq P_{i}, 1 \leq t_{i} \leq K_{i}\right\}\right|<\left|\left\{t_{i} \mid \hat{P}_{i}^{t_{i}} \neq P_{i}, 1 \leq t_{i} \leq K_{i}\right\}\right|
$$

We provide a necessary and sufficient condition of implementation by a finite mechanism under this sincerity ordering $(* *)$.

Suppose that an SCC F satisfies strong chain dominance. Theorem 1 states that this is equivalent to implementability by a bounded mechanism under sincerity ordering $\left(^{*}\right)$. Let $z$ be the function introduced in Definition 4. For each $i \in N$, each $t_{i} \in \mathbb{N}$, and each $C_{i} \in \mathcal{C}_{i}$, let $\left.z\right|_{i, t_{i}, C_{i}}: A \times \mathbb{N}^{n-1} \times \mathcal{C}_{-i} \rightarrow A$ be a function defined by $\left.z\right|_{i, t_{i}, C_{i}}\left(a, t_{-i}, C_{-i}\right)=$ $z\left(a,\left(t_{i}, t_{-i}\right),\left(C_{i}, C_{-i}\right)\right)$.

Proposition 2. A social choice correspondence $F$ is implemented in undominated strategies by a finite mechanism under sincerity ordering $\left(^{* *}\right)$ if and only if there exist a function $z$ and integer $L(<\infty)$ such that $z$ satisfies conditions (i)-(iv) in Definition 4 , and $\mid\left\{\left.z\right|_{i, t_{i}, C_{i}} ; t_{i} \in\right.$ $\mathbb{N}\} \mid \leq L$ for each $i \in N$ and each $C_{i} \in \mathcal{C}_{i}$.

Recall the function $z$ as described in the end of Section 2 when $F$ is top-inclusive. Since $\left.z\right|_{i, t_{i}, C_{i}}=\left.z\right|_{i, t_{i}^{\prime}, C_{i}}$ when $t_{i}, t_{i}^{\prime} \geq 2$ and $C_{i}\left(t_{i}\right)=C_{i}\left(t_{i}^{\prime}\right)$, this $z$ satisfies the condition in Proposition 2 with $L=2\left|\mathcal{D}_{i}\right|$. Thus, we showed the following statement:

Corollary 3. Suppose that all agents are partially honest with sincerity ordering (**). If an SCC F satisfies top-inclusiveness, then F is implemented in undominated strategies by a finite mechanism. 
In particular, the Pareto correspondence is implemented in undominated strategies by a finite mechanism. ${ }^{15}$

The construction of an implementing mechanism is essentially a special case of the proof of Theorem 1. We explain below how to construct a mechanism under top-inclusiveness in order to extract essences in the proof of Proposition 2 and Theorem 1. For each $i \in N$, let $M_{i}=\{1, \ldots,|A|\} \times \mathcal{D}_{i} \times \mathcal{D}_{i}$, in which for each $m_{i}=\left(\alpha_{i}, P_{i}^{1}, P_{i}^{2}\right) \in M_{i}$ and each $\hat{m}_{i}=$ $\left(\hat{\alpha}_{i}, \hat{P}_{i}^{1}, \hat{P}_{i}^{2}\right) \in M_{i}$, the sincerity ordering (**) is given by $m_{i} S_{i}^{P_{i}} \hat{m}_{i}$ if and only if either (i) $P_{i}^{1}=P_{i}^{2}=P_{i}$ and $\left(\hat{P}_{i}^{1} \neq P_{i}\right.$ or $\left.\hat{P}_{i}^{2} \neq P_{i}\right)$, or (ii) $\left(P_{i}^{1}=P_{i}\right.$ or $\left.P_{i}^{2}=P_{i}\right)$ and $\left(\hat{P}_{i}^{1} \neq P_{i}\right.$ and $\left.\hat{P}_{i}^{2} \neq P_{i}\right){ }^{16}$ For each $\left(\alpha_{i}\right)_{i \in N} \in\{1, \ldots,|A|\}^{n}$, let $\beta$ be an integer satisfying $1 \leq \beta \leq|A|$ and $^{17}$

$$
\beta \equiv\left(\sum_{i \in N} \alpha_{i}\right) \bmod |A| .
$$

We define the assignment function $g$ as follows:

$$
g\left(\left(\alpha_{i}, P_{i}^{\prime}, P_{i}\right)_{i \in N}\right)= \begin{cases}r^{\beta}\left(P_{1}, F(P)\right) & \text { if } P_{i}^{\prime}=P_{i} \text { for all } i \in N, \text { and } 1 \leq \beta \leq|F(P)|, \\ r^{1}\left(P_{1}, F(P)\right) & \text { if } P_{i}^{\prime}=P_{i} \text { for all } i \in N, \text { and }|F(P)|+1 \leq \beta \leq|A|, \\ r^{1}\left(P_{j}, A\right) & \text { otherwise, where } j=\min \left\{i \in N \mid P_{i} \neq P_{i}^{\prime}\right\} .\end{cases}
$$

In this mechanism, the preferences $P_{i}^{\prime}$ and $P_{i}$ represent $i$ 's reports on her preference. The mechanism designer regards the latter preference $P_{i}$ as $i$ 's true preference. Thus, $P_{i}^{\prime} \neq P_{i}$ immediately implies that agent $i$ makes a false report. If at least one agent is apparently lying, the implemented alternative is the top one with respect to the true preference of the lying agent with the smallest index. An agent $i$ can implement her best alternative at her true preference $P_{i}$ by reporting a pair of distinct messages $\left(P_{i}^{\prime}, P_{i}\right)\left(P_{i}^{\prime} \neq P_{i}\right)$ whenever no other agents do. If every agent $i$ makes a report $\left(\alpha_{i}, P_{i}^{\prime}, P_{i}\right)$ with $P_{i}^{\prime}=P_{i}$, the mechanism determines an alternative according to a modulo procedure. ${ }^{18}$ Because of this modulo structure, for each

\footnotetext{
${ }^{15}$ The Pareto correspondence selects for each $P \in \mathcal{D}$, all alternatives $a \in A$ such that there does not exist another alternative $b \in A$ with $b P_{i} a$ for all $i \in N$.

${ }^{16}$ In this case, we can show that the same mechanism implements $F$ in undominated strategies under a simpler sincerity ordering $\tilde{S}_{i}^{P_{i}}$ defined as $m_{i} \tilde{S}_{i}^{P_{i}} \hat{m}_{i}$ if and only if $P_{i}^{1}=P_{i}^{2}=P_{i}$ and $\left(\hat{P}_{i}^{1} \neq P_{i}\right.$ or $\left.\hat{P}_{i}^{2} \neq P_{i}\right)$. Since this ordering divides the message space into "sincere messages" with $P_{i}^{1}=P_{i}^{2}=P_{i}$ and other "insincere messages," a top-inclusive SCC is implemented in undominated strategies also when partial honesty is defined as in Dutta and Sen (2012) (see footnote 5 for relations to their definition of partial honesty).

${ }^{17}$ Note that $p \equiv r \bmod q$ if and only if $p-r$ is a multiple of $q$.

${ }^{18}$ One might wonder why this modulo structure is different from modulo games in the literature on Nash implementation, in which each agent $i$ calls an index $\alpha_{i}$ in $N$, and the winner $j$ is determined by $j=\sum_{i \in N} \alpha_{i}$ mod $n$ who can choose the most-preferred among permissive alternatives. We do not adopt such a modulo game because this game may not implement the alternatives that are not top-ranked for any agent. Recall that our purpose of adopting the modulo structure is quite different from Nash implementation, as we noted in footnote 14 .
} 
$P \in \mathcal{D}$, each $a \in F(P)$, each $i \in N$, and each message $m_{j}=\left(\alpha_{j}, P_{j}, P_{j}\right)$ of each agent $j \neq i$, there exists $i$ 's message $m_{i}=\left(\alpha_{i}, P_{i}, P_{i}\right)$ such that $\left(m_{i}, m_{-i}\right)$ implements $a$.

The above finite mechanism $(M, g)$ implements the top-inclusive SCC $F$ in undominated strategies under the sincerity ordering $(* *)$. Although the proof is a special case of Proposition 2, we provide an explicit proof in order to present the method employed in our construction of the implementing mechanism.

First, we show that every alternative in $F(P)$ is implemented in undominated strategies at each $P \in \mathcal{D}$. To show this, we prove that for each $i \in N$, each $\alpha_{i} \in\{1, \ldots,|A|\}$, and each $P_{i} \in \mathcal{D}_{i}$, message $\left(\alpha_{i}, P_{i}, P_{i}\right) \in M_{i}$ must be undominated at $P_{i}$. By the construction of the mechanism, there exists $\left(\alpha_{j}, P_{j}, P_{j}\right)_{j \in N \backslash\{i\}} \in M_{-i}$ such that the implemented alternative is $r^{1}\left(P_{i}, F(P)\right)$. This is the top alternative $r^{1}\left(P_{i}, A\right)$ because of top-inclusiveness. Since no message is more sincere than $\left(\alpha_{i}, P_{i}, P_{i}\right)$ at $P_{i}$, message $\left(\alpha_{i}, P_{i}, P_{i}\right)$ is undominated at $P_{i}$. (It is easy to see that for each $\alpha_{i}, \alpha_{i}^{\prime}$, there is no dominance relation between $\left(\alpha_{i}, P_{i}, P_{i}\right)$ and $\left(\alpha_{i}^{\prime}, P_{i}, P_{i}\right)$ at $\left.P_{i}.\right)$ Therefore, for each $P \in \mathcal{D}$, every alternative in $F(P)=\{g(\alpha, P, P) \mid \alpha \in$ $\left.\{1, \ldots,|A|\}^{n}\right\}$ is implemented in undominated strategies at $P$.

Second, we show that no alternative in $A \backslash F(P)$ is implemented in undominated strategies at each $P \in \mathcal{D}$. By top-inclusiveness of $F$ and by the construction of the mechanism, for each $P \in \mathcal{D}$ and each $m=\left(\alpha_{i}, P_{i}^{\prime}, P_{i}\right)_{i \in N}$, we have $g(m) \in F(P)$. By the definition of the sincerity ordering, for each $i \in N$, each $P_{i} \in \mathcal{D}_{i}$ and each $\tilde{m}_{i}=\left(\alpha_{i}, \tilde{P}_{i}^{\prime}, \tilde{P}_{i}\right) \in M_{i}, \tilde{m}_{i}$ is not more sincere than $m_{i}=\left(\alpha_{i}, P_{i}^{\prime}, P_{i}\right) \in M_{i}$ at $P_{i}$ whenever $\tilde{P}_{i} \neq P_{i}$. Therefore, it

suffices to show that for each $i \in N$, each $P_{i} \in \mathcal{D}_{i}$ and each $\tilde{m}_{i}=\left(\alpha_{i}, \tilde{P}_{i}^{\prime}, \tilde{P}_{i}\right) \in M_{i}$ with $\tilde{P}_{i} \neq P_{i}$, there exists a message $m_{i}=\left(\alpha_{i}, P_{i}^{\prime}, P_{i}\right) \in M_{i}$ such that $g\left(m_{i}, m_{-i}\right) R_{i} g\left(\tilde{m}_{i}, m_{-i}\right)$ for all $m_{-i} \in M_{-i}$. By the construction of the mechanism, we have $g\left(m_{i}, m_{-i}\right)=g\left(\tilde{m}_{i}, m_{-i}\right)$ whenever some agent with an index smaller than $i$ is lying in $m_{-i}$. Thus, it suffices to consider $m_{-i} \in M_{-i}$ in which no agent with a smaller index is lying. Let $m_{i}=\left(\alpha_{i}, \tilde{P}_{i}, P_{i}\right) \in M_{i}$. Since we assumed $\tilde{P}_{i} \neq P_{i}$ and no other agent is lying, $g\left(m_{i}, m_{-i}\right)$ is the top alternative at $P_{i}$, and $g\left(m_{i}, m_{-i}\right) R_{i} g\left(\tilde{m}_{i}, m_{-i}\right)$ follows immediately. Hence, $g\left(m_{i}, m_{-i}\right) R_{i} g\left(\tilde{m}_{i}, m_{-i}\right)$ for all $m_{-i} \in M_{-i}$.

\subsection{Examples}

Strong chain dominance is a property of SCCs. We end this section by giving examples of SCCs satisfying this property and of SCCs failing to satisfy it. To proceed, we define these SCCs. The tops-only correspondence selects for each $P \in \mathcal{D}$, all alternatives $a \in A$ that are top alternatives for some $i \in N$, i.e. $\bigcup_{i \in N}\left\{r^{1}\left(P_{i}, A\right)\right\}$. A positional scoring SCC is given by a scoring vector $s=\left(s_{1}, s_{2}, \ldots, s_{|A|}\right) \in \mathbb{R}^{|A|}$ with $s_{1} \geq s_{2} \geq \ldots \geq s_{|A|}$ and $s_{1}>s_{|A|}$, where $s_{k}$ 
is the points awarded to the $k$ th ranked alternative. Positional scoring SCC $F$ for $s$ selects all alternatives with the highest total points, i.e. for each $P \in \mathcal{D}, a \in F(P)$ if and only if for each $b \in A, \sum_{k \in\{1,2, \ldots|A|\}} s_{k}\left|\left\{i \in N \mid r^{k}\left(P_{i}, A\right)=a\right\}\right| \geq \sum_{k \in\{1,2, \ldots|A|\}} s_{k}\left|\left\{i \in N \mid r^{k}\left(P_{i}, A\right)=b\right\}\right|$. The plurality correspondence has $s=(1,0, \ldots, 0)$ and Borda's method, $(|A|-1,|A|-2, \ldots, 0)$.

The tops-only correspondence satisfies top-inclusiveness, hence it satisfies strong chain dominance. The plurality correspondence satisfies it if $n=2$ or $|A|=2$. One can see that when $n=2$, these SCCs coincide. When $n>2$, they do not coincide, hence the plurality correspondence does not satisfy top-inclusiveness. When $|A|=2$, it satisfies strong chain dominance. As stated after Definition 5, top-inclusiveness is thus stronger than strong chain dominance. Also when $|A|=2$, any other positional scoring SCC, including Borda's method, satisfies strong chain dominance. The plurality correspondence however does not satisfy it if $n>2$ and $|A|>2$, nor does any other positional scoring SCC, including Borda's method, if $|A|>2$.

By Theorem 1, strong chain dominance is necessary and sufficient for implementation by a bounded mechanism when all agents are partially honest with sincerity. Thus, SCCs satisfying this property are implementable, whereas those failing to satisfy it are not implementable. Further, by Corollary 3, top-inclusiveness is sufficient for implementation by a finite mechanism. The following three corollaries therefore follow. We postpone the proof of Corollary 6 to Appendix A.3.

Corollary 4. The tops-only correspondence is implementable in undominated strategies by a finite mechanism.

Proof. The tops-only correspondence satisfies top-inclusiveness. By Corollary 3 , the statement is immediate.

Corollary 5. The plurality correspondence is implementable in undominated strategies by a bounded mechanism if $n=2$ or $|A|=2$. Any other positional scoring SCC, including Borda's method, is implementable in undominated strategies by a bounded mechanism if $|A|=2$.

Proof. When $n=2$, the plurality correspondence coincides with the tops-only correspondence, hence it satisfies strong chain dominance. When $|A|=2$, by taking $z(a, t, C)=a$ if $t=(1, \ldots, 1)$, and $z(a, t, C)=r^{1}\left(C_{j}\left(t_{j}\right), A\right)$ otherwise, where $j=\min \left\{i \in N \mid t_{i}>1\right.$ and $\left.r^{1}\left(C_{i}\left(t_{i}\right), A\right) \in F(C(t))\right\}$, one can see that conditions (i)-(iv) in Definition 4 are satisfied, hence the plurality correspondence satisfies strong chain dominance.

When $|A|=2$, any other positional scoring SCC, including Borda's method coincides with the plurality correspondence, hence it satisfies strong chain dominance. 
Corollary 6. The plurality correspondence is not implementable in undominated strategies by a bounded mechanism if $n>2$ and $|A|>2$. Any other positional scoring $S C C$, including Borda's method, is not implementable in undominated strategies by a bounded mechanism if $|A|>2$.

Remark 4. In Appendix A.1.1, we prove that strong chain dominance is necessary for implementation by a bounded mechanism with respect to material preferences. Thus, as stated in Proposition 7 in Section 4 below, strong chain dominance is necessary under any sincerity ordering. Corollary 6 is therefore general in that sense.

Remark 5. Börgers (1991) provides a bounded mechanism that implements the tops-only correspondence with respect to material preferences. Ohseto (1994) provides a bounded mechanism derived from this mechanism that implements the plurality correspondence with respect to material preferences if $n=2$ or $|A|=2$. By Corollary 8 , these correspondences are implementable by a bounded mechanism under any sincerity ordering, in particular under the sincerity ordering $(*)$. The logic of our proofs is different, as we prove satisfaction of a necessary and sufficient condition.

Ohseto (1994) proves by contradiction that the plurality correspondence is not implementable with respect to material preferences if $n>2$ and $|A|>2$. As a SCC may fail to be implementable by a bounded with respect to material preferences, but implementable under partial honesty, as later proven in Example 1, this result does not imply Corollary 6. However, as noted in the above remark, the converse holds.

Remark 6. Ohseto (1994) proves that the plurality correspondence satisfies strategyresistance if and only if $n$ and $|A|$ satisfy one of the following conditions: (i) $n=2$, (ii) $|A|=2$, (iii) $n=3$ and $|A|>2$, (iv) $n=5$ and $|A|=3$. In Appendix A.3, we prove that this SCC fails to satisfy strong chain dominance if $n>2$ and $|A|>2$. Thus, there is a failure of this condition if condition (iii) or (iv) holds. This illustrates in which sense strong chain dominance is stronger than strategy-resistance.

Finally, we show that if agents are not partially honest, strong chain dominance is not a sufficient condition for implementation in undominated strategies by a bounded mechanism with respect to material preferences. In the following example, we provide a SCC which satisfies strong chain dominance (thus implementable in undominated strategies by a bounded mechanism when agents are partially honest), but is not implementable in undominated strategies by a bounded mechanism with respect to material preferences.

Example 1. Let $n=2$ and $A=\{a, b, c\}$. Let $P_{1}$ satisfy a $P_{1} c P_{1} b, P_{1}^{\prime}$ satisfy $a P_{1}^{\prime} b P_{1}^{\prime} c$, and $P_{2}$ satisfy $b P_{2} c P_{2} a$. In restricted domain $\mathcal{D}=\left\{P_{1}, P_{1}^{\prime}\right\} \times\left\{P_{2}\right\}$, let a SCC $F$ be such 
that $F\left(P_{1}, P_{2}\right)=\{a\}$ and $F\left(P_{1}^{\prime}, P_{2}\right)=\{a, b\}$. This SCC $F$ satisfies strong chain dominance, but is not implementable in undominated strategies by a bounded mechanism if all agents only care about material preferences.

First, we show that $F$ satisfies strong chain dominance by constructing a function $z$ satisfying conditions (i)-(iv) in Definition 4. For each $t \in \mathbb{N}^{2}$ and each $C \in \mathcal{C}$, let $z(a, t, C)=$ a. Also, for each $t \in \mathbb{N}^{2}$ and each $C \in \mathcal{C}$ with $C_{1}(1)=P_{i}^{\prime}$, let $z(b, t, C)=b$ if $t_{1}=1$, and $z(b, t, C)=a$ if $t_{1} \geq 2$. It is easy to check that this function $z$ satisfies conditions (i)-(iv) in Definition 4.

Second, we show that $F$ is not implementable in undominated strategies by a bounded mechanism if all agents only care about material preferences. Assume on the contrary that a bounded mechanism $(M, g)$ implements $F$ in undominated strategies. There exists a message $\bar{m}_{1} \in M_{1}$ such that $\bar{m}_{1}$ is undominated at $P_{1}$. Let $M_{2}^{\prime} \subset M_{2}$ be the subset of messages $m_{2}^{\prime}$ such that $g\left(\bar{m}_{1}, m_{2}^{\prime}\right) \neq a$. Since $F\left(P_{1}, P_{2}\right)=\{a\}$, for each $m_{2}^{\prime} \in M_{2}^{\prime}, m_{2}^{\prime}$ is dominated at $P_{2}$. By boundedness, for each $m_{2}^{\prime} \in M_{2}^{\prime}$, there exists $\bar{m}_{2} \in M_{2}$ which is undominated and dominates $m_{2}^{\prime}$ at $P_{2}$. Since $\bar{m}_{2}$ is undominated at $P_{2}, \bar{m}_{2} \in M_{2} \backslash M_{2}^{\prime}$. However, because $a$ is the least-preferred alternative at $P_{2}$, for each $m_{2}^{\prime} \in M_{2}^{\prime}, g\left(\bar{m}_{1}, m_{2}^{\prime}\right) P_{2} a=g\left(\bar{m}_{1}, \bar{m}_{2}\right)$. This implies that $M_{2}^{\prime}=\emptyset$, that is, $g\left(\bar{m}_{1}, m_{2}\right)=a$ for all $m_{2} \in M_{2}$. Thus, for each $m_{1} \in M_{1}, \bar{m}_{1}$ dominates $m_{1}$ at $P_{1}^{\prime}$ as well as at $P_{1}$ unless $g\left(m_{1}, m_{2}\right)=a$ for all $m_{2} \in M_{2}$. Hence, if $m_{1}$ is undominated at $P_{1}^{\prime}, g\left(m_{1}, m_{2}\right)=a$ for all $m_{2} \in M_{2}$. This contradicts $b \in F\left(P_{1}^{\prime}, P_{2}\right)$.

\section{Other Sincerity Orderings}

In Theorem 1, we considered the sincerity ordering $\left(^{*}\right)$ where the message space is $M_{i} \subseteq$ $\Sigma_{i} \times \mathcal{D}_{i} \times \mathcal{D}_{i} \times \cdots$ for each $i \in N$ with $\Sigma_{i}$ being a certain finite or countable set. In this section, we argue that the necessary and sufficient condition in Theorem 1 is true under many other sincerity orderings.

\subsection{Necessary Condition}

The necessity part is easier. In fact, the result does not depend on details of sincerity orderings.

Proposition 7. Under any sincerity ordering in any bounded mechanism $\Gamma$, strong chain dominance is a necessary condition of implementability in undominated strategies.

We show this general statement in the proof of necessity in Appendix A.1.1.

As a trivial case in which all messages are equally sincere, Proposition 7 shows that strong chain dominance is a necessary condition of implementability in undominated strategies by 
a bounded mechanism with respect to material preferences. Together with the sufficiency part of Theorem 1, we obtained the following:

Corollary 8. Strong chain dominance is a necessary condition of implementability in undominated strategies by a bounded mechanism when all agents only care about material preferences. Therefore, an SCCF is implementable in undominated strategies by a bounded mechanism when no agent is partially honest, then $F$ is implementable in undominated strategies by a bounded mechanism when all agents are partially honest under the sincerity ordering $(*)$.

Therefore, when agents are partially honest, SCCs are more likely to be implementable in undominated strategies by bounded mechanisms.

\subsection{Sufficient Condition}

We discussed in Section 3.1 that there are two kinds of difficulties in constructing an implementing mechanism, and partial honesty resolves these difficulties. In this section, we argue that if a sincerity ordering satisfies certain conditions, the mechanism we construct in the proof of Theorem 1 also implements the SCC under the sincerity ordering. Let $(M, g)$ be the mechanism in which $M_{i} \subseteq \Sigma_{i} \times \mathcal{D}_{i} \times \mathcal{D}_{i} \times \ldots$ where $\Sigma_{i}$ is a finite or countable set for each $i \in N$. For each $i \in N$ and each $K_{i} \in \mathbb{N}$, let $\tilde{\mathcal{C}}_{i}^{K_{i}} \subset \mathcal{C}_{i}$ be a subset of chains $C_{i} \in \mathcal{C}_{i}$ such that $C_{i}\left(t_{i}\right) \neq C_{i}\left(t_{i}+1\right)$ for each $t_{i}<K_{i}$, and $C_{i}\left(t_{i}\right)=C_{i}\left(K_{i}\right)$ for each $t_{i} \geq K_{i}$. Let $\tilde{\mathcal{C}}_{i}=\bigcup_{K_{i}=1}^{\infty} \tilde{\mathcal{C}}_{i}^{K_{i}}$.

Proposition 9. Suppose that a sincerity ordering $S_{i}^{P_{i}}$ satisfies the following four conditions: for each $i \in N$, each $P_{i} \in \mathcal{D}_{i}$, and each $m_{i}=\left(\sigma_{i}, P_{i}^{1}, P_{i}^{2}, \ldots\right) \in M_{i}$,

(a) $\hat{m}_{i} I\left(S_{i}^{P_{i}}\right) m_{i}$ for each $\hat{m}_{i}=\left(\hat{\sigma}_{i}, P_{i}^{1}, P_{i}^{2}, \ldots\right) \in M_{i}$,

(b) if there exists $t_{i}$ such that $P_{i}^{t_{i}} \neq P_{i}$, then $\hat{m}_{i} S_{i}^{P_{i}} m_{i}$ where $\hat{m}_{i}=\left(\sigma_{i}, P_{i}, P_{i}, P_{i}, \ldots\right) \in M_{i}$,

(c) for each $\hat{m}_{i}=\left(\sigma_{i}, \hat{P}_{i}^{1}, \hat{P}_{i}^{2}, \ldots\right) \in M_{i}$, if there exists $\bar{t}_{i}$ such that $\hat{P}_{i}^{t_{i}}=P_{i}^{t_{i}}$ for each $t_{i} \leq \bar{t}_{i}-1$, and $\hat{P}_{i}^{t_{i}}=P_{i}$ for each $t_{i} \geq \bar{t}_{i}$, then $\hat{m}_{i} S_{i}^{P_{i}} m_{i}$ or $\hat{m}_{i} I\left(S_{i}^{P_{i}}\right) m_{i}$, and

(d) if there exists $\bar{t}_{i}$ such that $P_{i}^{t_{i}}=P_{i}$ for all $t_{i} \geq \bar{t}_{i}$, then the set of messages $\left\{\left(\sigma_{i}, \hat{C}_{i}\right) \in\right.$ $M_{i} \mid \hat{C}_{i} \in \tilde{\mathcal{C}}_{i}$, and $\left(\left(\sigma_{i}, \hat{C}_{i}\right) S_{i}^{P_{i}} m_{i}\right.$ or $\left.\left.\left(\sigma_{i}, \hat{C}_{i}\right) I\left(S_{i}^{P_{i}}\right) m_{i}\right)\right\}$ is finite.

Then, strong chain dominance is a sufficient condition of implementability in undominated strategies by a bounded mechanism under this sincerity ordering. 
Condition (a) is our assumption that $\Sigma_{i}$ is the part of the message irrelevant to the sincerity of agent $i$. The messages are equally sincere if they have the same chain $\left(P_{i}^{1}, P_{i}^{2}, \ldots\right)$. Condition (b) says that a fully sincere message that never tells a lie should be more sincere than messages of any other forms. Condition (c) is a natural requirement saying that a message becomes weakly more sincere if the agent alters the tail of the chain to report the true preference. Condition (d) guarantees boundedness in our construction of the mechanism. This condition (d) says that if $m_{i}$ reports false preferences only finite times, then $m_{i}$ is more sincere than all messages with chains in $\tilde{\mathcal{C}}_{i}$ with only finite exceptions.

It is easily seen that sincerity ordering $\left(^{*}\right)$ satisfies all conditions in Proposition 9. Therefore, the sufficiency part of Theorem 1 is a corollary of Proposition 9.

Lemma 10. Sincerity ordering (*) satisfies all conditions (a)-(d) in Proposition 9.

Proof. It clearly satisfies conditions (a), (b), and (c). We show that sincerity ordering $\left(^{*}\right)$ satisfies condition (d) in Proposition 9. Let $L$ be the number of times a message $m_{i}$ tells a lie at $P_{i} \in \mathcal{D}_{i}$. Suppose that a message $\hat{m}_{i}$ has a chain $\left(\hat{P}_{i}^{1}, \hat{P}_{i}^{2}, \ldots\right) \in \tilde{\mathcal{C}}_{i}^{K_{i}}$. Since $\hat{P}_{i}^{t_{i}} \neq \hat{P}_{i}^{t_{i}+1}$ for each $t_{i}$ with $1 \leq t_{i} \leq K_{i}-1, \hat{m}_{i}$ reports a false preference more than $L$ times if $K_{i} \geq 2(L+1)$. Since there are only finite messages in $\bigcup_{K_{i}=1}^{2 L+1} \tilde{\mathcal{C}}_{i}^{K_{i}}$, condition (d) is satisfied.

Our construction of the implementing mechanism is based on the following idea of recursive definitions: We start with a mechanism with a modulo structure which may implement both desired and undesired alternatives. Condition (b) guarantees that each message $\left(\sigma_{i}, P_{i}, P_{i}, \ldots\right)$ in this initial mechanism is undominated at the reported preference $P_{i}$, and all the alternatives assigned by the SCC are implemented. In the subsequent steps, we add messages that dominate the messages in the previous step which may implement undesired alternatives. Condition (c) guarantees that the added message $m_{i}$ is not more sincere than the message $\hat{m}_{i}$ which exists in the previous steps, at the preference reported infinitely in the tail of $\hat{m}_{i}$. Thus this additional process does not generate dominance relations we do not want. This process continues infinitely. Finally, we have to show boundedness of the mechanism. If the mechanism is unbounded, there exists an infinite sequence of messages in which the $k$ th message is dominated by the $k^{\prime}$ th message for each $k<k^{\prime}$. This cannot happen because condition $(\mathrm{d})$ implies that the $k$ th message is more sincere than any other messages with finite exceptions, and so the number of the messages dominating the $k$ th message must be finite.

Combined with Proposition 7, we have shown the following:

Corollary 11. Suppose that the sincerity ordering satisfies conditions (a)-(d) in Proposition 9. Then, strong chain dominance is a necessary and sufficient condition of implementability in undominated strategies by a bounded mechanism. 
There are other sincerity orderings that satisfy conditions (a)-(d) in Proposition 9. For example, a sincerity ordering $\hat{S}_{i}^{P_{i}}$ defined as for each $m_{i}=\left(\sigma_{i}, P_{i}^{1}, P_{i}^{2}, \ldots\right)$ and each $\hat{m}_{i}=\left(\hat{\sigma}_{i}, \hat{P}_{i}^{1}, \hat{P}_{i}^{2}, \ldots\right), m_{i} \hat{S}_{i}^{P_{i}} \hat{m}_{i}$ if and only if $\max \left\{t_{i} \mid P_{i}^{t_{i}} \neq P_{i}\right\}<\max \left\{t_{i} \mid \hat{P}_{i}^{t_{i}} \neq P_{i}\right\} .{ }^{19}$ This ordering may be interpreted as a situation in which an agent reports her preference repeatedly, and she is more sincere if she becomes truthful earlier, where it is considered that she is truthful at time $t_{i}$ if she reports the true preference after $t_{i}$ onward.

We conclude this section by noting a simple sincerity ordering $\bar{S}_{i}^{P_{i}}$ defined as, for each $m_{i}=\left(\sigma_{i}, P_{i}^{1}, P_{i}^{2}, \ldots\right)$ and each $\hat{m}_{i}=\left(\hat{\sigma}_{i}, \hat{P}_{i}^{1}, \hat{P}_{i}^{2}, \ldots\right), m_{i} \bar{S}_{i}^{P_{i}} \hat{m}_{i}$ if and only if $P_{i}=P_{i}^{1}=$ $P_{i}^{2}=\cdots$ and there exists $k$ such that $\hat{P}_{i}^{k} \neq P_{i}$. For each preference $P_{i}$, this ordering divides the message space into the set of fully sincere messages $\left(\sigma_{i}, P_{i}, P_{i}, \ldots\right)$, and the remainder. This is the situation considered by Dutta and Sen (2012), in which there are no messages with "middle" sincerity, and all messages are classified into two sets; fully sincere messages and completely insincere messages. This sincerity ordering satisfies conditions (a)-(c), but does not satisfy condition (d) because there are no messages with "middle" sincerity. However, if the condition in Proposition 2 is satisfied, then finiteness of the mechanism is immediately guaranteed, and condition (d) is dispensable because this condition is applied in the proof of boundedness of the mechanism. Therefore, Proposition 2 holds true under this sincerity ordering $\bar{S}_{i}^{P_{i}}$ instead of sincerity ordering (**).

\section{Conclusion}

In this paper we have considered a weak solution concept of implementation theoryimplementation in undominated strategies by a bounded mechanism. We follow the same set-up as in Jackson (1992) which introduced this notion of implementation, and in addition we assume that the agents are partially honest. Thus the agents have a preference over messages in a lexicographic manner - if two different messages for any agent produce the same outcome against some message profile of the other agents, then she strictly prefers the message that is "more sincere." This provides another parameter to compare the dominance relations between messages for an agent. We completely characterize the class of SCCs that are implementable in undominated strategies by a bounded mechanism. In an important application, we show that the Pareto correspondence is implementable by a finite mechanism.

\footnotetext{
${ }^{19}$ For a set $X \subseteq \mathbb{N}$, we regard $\max X=\infty$ if $X$ has no maximum element. By rule, $\infty=\infty$, and $m<\infty$ for any finite number $m$.
} 


\section{A Appendix: Proofs of the Results}

\section{A.1 Proof of Theorem 1}

Suppose that a mechanism $(M, g)$ is considered. We say that agent $i$ 's message $m_{i} \in M_{i}$ very weakly dominates another message $m_{i}^{\prime} \in M_{i}$ at $P_{i} \in \mathcal{D}_{i}$ with respect to material preferences if $g\left(m_{i}, m_{-i}\right) R_{i} g\left(m_{i}^{\prime}, m_{-i}\right)$ for all $m_{-i} \in M_{-i}$. We also say that a message $m_{i} \in M_{i}$ very weakly dominates another message $m_{i}^{\prime} \in M_{i}$ at $P_{i} \in \mathcal{D}_{i}$ (with respect to preferences with partial honesty) if $\left(m_{i}, m_{-i}\right) \succsim_{i}^{P_{i}}\left(m_{i}^{\prime}, m_{-i}\right)$ for all $m_{-i} \in M_{-i}$. By the definition of partial honesty, if $m_{i}$ very weakly dominates $m_{i}^{\prime}$ at $P_{i} \in \mathcal{D}_{i}$ in a mechanism $(M, g)$ (with respect to preferences with partial honesty), then $m_{i}$ very weakly dominates $m_{i}^{\prime}$ at $P_{i}$ with respect to material preferences (but not vice versa).

\section{A.1.1 Proof of Necessity of Theorem 1}

We show Proposition 7, which is stronger than the necessity of Theorem 1. I.e., strong chain dominance is the necessary condition under an arbitrary sincerity ordering $S_{i}^{P_{i}}$ in any bounded mechanism $(M, g)$. We show that in this general environment, $F$ satisfies strong chain dominance if an SCC $F$ is implemented in undominated strategies by a bounded mechanism.

Suppose that a bounded mechanism $(M, g)$ implements $F$ in undominated strategies. For each $i \in N$, each $P_{i} \in \mathcal{D}_{i}$, and each $m_{i} \in M_{i}$, there exists an undominated message $m_{i}^{\prime} \in U_{i}\left(P_{i}\right)$ which very weakly dominates $m_{i}$ at $P_{i} .{ }^{20}$ For each $i \in N$ and each $P_{i} \in \mathcal{D}_{i}$, we fix a function $d_{i}^{P_{i}}: M_{i} \rightarrow M_{i}$ such that for each $m_{i} \in M_{i}, d_{i}^{P_{i}}\left(m_{i}\right)$ is undominated and very weakly dominates $m_{i}$ at $P_{i}$.

For each $\bar{P} \in \mathcal{D}$ and each $a \in F(\bar{P})$, let $\bar{m}(a, \bar{P}) \in M$ be an arbitrarily chosen message profile such that $g(\bar{m}(a, \bar{P}))=a$ and $\bar{m}_{i}(a, \bar{P})$ is undominated at $\bar{P}_{i}$ for each $i \in N$. Such a message profile exists because $F$ is implemented in undominated strategies by $(M, g)$, and $a \in F(\bar{P})$. For each $i \in N$, each $\bar{P} \in \mathcal{D}$, each $a \in F(\bar{P})$, and each $C_{i} \in \mathcal{C}_{i}$ with $C_{i}(1)=\bar{P}_{i}$, we recursively define an infinite sequence of messages $m_{i}^{1}\left(a, \bar{P}, C_{i}\right), m_{i}^{2}\left(a, \bar{P}, C_{i}\right), \ldots$ as follows: First, let $m_{i}^{1}\left(a, \bar{P}, C_{i}\right)=\bar{m}_{i}(a, \bar{P})$. Next, when

$m_{i}^{k-1}\left(a, \bar{P}, C_{i}\right)$ is given for $k \geq 2$, let $m_{i}^{k}\left(a, \bar{P}, C_{i}\right)=d_{i}^{C_{i}(k)}\left(m_{i}^{k-1}\left(a, \bar{P}, C_{i}\right)\right)$. This is the undominated message which very weakly dominates $m_{i}^{k-1}\left(a, \bar{P}, C_{i}\right)$ at $C_{i}(k)$. If $a \notin F(\bar{P})$, we fix a sequence $m_{i}^{1}\left(a, \bar{P}, C_{i}\right), m_{i}^{2}\left(a, \bar{P}, C_{i}\right), \ldots$ arbitrarily. (Since strong chain dominance imposes no requirement on $z(a, t, C)$ whenever $a \notin F\left(C_{1}(1), \ldots, C_{n}(1)\right)$, this sequence can

\footnotetext{
${ }^{20}$ If $m_{i}$ is dominated at $P_{i}$, then by boundedness of the mechanism, there exists an undominated message $m_{i}^{\prime} \in U_{i}\left(P_{i}\right)$ which dominates $m_{i}$ at $P_{i}$. If $m_{i}$ is undominated at $P_{i}, m_{i}$ is very weakly dominated by itself at $P_{i}$ in a trivial sense.
} 
be anything.)

Now, we construct function $z(a, t, C)$, and show that $z$ satisfies conditions (i)-(iv) in Definition 4. For each $a \in A$, each $t \in \mathbb{N}^{n}$, and each $C \in \mathcal{C}$, we define $z(a, t, C)=$ $g\left(m_{1}^{t_{1}}\left(a, \bar{P}, C_{1}\right), \ldots, m_{n}^{t_{n}}\left(a, \bar{P}, C_{n}\right)\right)$, where $\bar{P}=\left(C_{1}(1), \ldots, C_{n}(1)\right)$. Condition (i) in Definition 4 holds because $m_{i}^{t_{i}}\left(a, \bar{P}, C_{i}\right)$ is undominated at $C_{i}\left(t_{i}\right)$ for each $i \in N$ and each $t_{i} \in \mathbb{N}$, and $F$ is implemented in undominated strategies by $(M, g)$. Since $z(a,(1, \ldots, 1), C)=$ $g\left(m_{1}^{1}\left(a, \bar{P}, C_{1}\right), \ldots, m_{n}^{1}\left(a, \bar{P}, C_{n}\right)\right)=g(\bar{m}(a, \bar{P}))=a$, condition (ii) in Definition 4 holds. Since the above construction of $z(a, t, C)$ is independent of $C_{i}\left(\tau_{i}\right)$ with $\tau_{i} \geq t_{i}+1$ for each $i \in N$, condition (iii) in Definition 4 holds. Since $m_{i}^{t_{i}}\left(a, \bar{P}, C_{i}\right)$ very weakly dominates $m_{i}^{t_{i}-1}\left(a, \bar{P}, C_{i}\right)$ at $C_{i}\left(t_{i}\right)$ for each $t_{i} \geq 2$, the definition of partial honesty implies that $m_{i}^{t_{i}}\left(a, \bar{P}, C_{i}\right)$ very weakly dominates $m_{i}^{t_{i}-1}\left(a, \bar{P}, C_{i}\right)$ at $C_{i}\left(t_{i}\right)$ with respect to material preferences for each $t_{i} \geq 2$. Thus, condition (iv) in Definition 4 is satisfied. Hence, $F$ satisfies strong chain dominance.

\section{A.1.2 Proof of Sufficiency of Theorem 1}

We show Proposition 9, which is stronger than the sufficiency of Theorem 1, as noted in Lemma 10. We construct a bounded mechanism that implements $F$ in undominated strategies under partial honesty, where the sincerity ordering satisfies conditions (a)-(d) in Proposition 9. Suppose that an SCC $F$ satisfies strong chain dominance, and let $z$ be the function introduced in Definition 4. The proof consists of four steps.

SteP 1: We construct a mechanism $(M, g)$.

Recall that for each $i \in N$ and each $K_{i} \in \mathbb{N}, \tilde{\mathcal{C}}_{i}^{K_{i}} \subset \mathcal{C}_{i}$ is a subset of chains such that $C_{i}\left(t_{i}\right) \neq C_{i}\left(t_{i}+1\right)$ for each $t_{i}<K_{i}$, and $C_{i}\left(t_{i}\right)=C_{i}\left(K_{i}\right)$ for each $t_{i} \geq K_{i}$, and $\tilde{\mathcal{C}}_{i}=\bigcup_{K_{i} \in \mathbb{N}} \tilde{\mathcal{C}}_{i}^{K_{i}}$. Let $\tilde{\mathcal{C}}=\prod_{i \in N} \tilde{\mathcal{C}}_{i}$.

Let $M_{i}=\{1, \ldots,|A|\} \times \mathbb{N} \times \tilde{\mathcal{C}}_{i}$ be the set of messages of each $i \in N$. For each $\alpha=$ $\left(\alpha_{1}, \ldots, \alpha_{n}\right) \in\{1, \ldots,|A|\}^{n}$, let $\beta$ be an integer satisfying $1 \leq \beta \leq|A|$ and $\beta \equiv\left(\sum_{i \in N} \alpha_{i}\right)$ $\bmod |A|$, and let

$$
a_{\alpha}= \begin{cases}r^{\beta}\left(C_{1}(1), F\left(C_{1}(1), \ldots, C_{n}(1)\right)\right) & \text { if } 1 \leq \beta \leq\left|F\left(C_{1}(1), \ldots, C_{n}(1)\right)\right|, \\ r^{1}\left(C_{1}(1), F\left(C_{1}(1), \ldots, C_{n}(1)\right)\right) & \text { otherwise. }{ }^{21}\end{cases}
$$

The assignment function $g: M \rightarrow A$ is defined as, for each $\alpha=\left(\alpha_{1}, \ldots, \alpha_{n}\right) \in\{1, \ldots,|A|\}^{n}$,

\footnotetext{
${ }^{21}$ Recall that we denote the $k$ th ranked alternative among $A^{\prime} \subseteq A$ for an agent at $P_{i}$ by $r^{k}\left(P_{i}, A^{\prime}\right) \in A^{\prime}$. Thus, for each $C \in \tilde{\mathcal{C}}, r^{\beta}\left(C_{1}(1), F\left(C_{1}(1), \ldots, C_{n}(1)\right)\right)$ and $r^{1}\left(C_{1}(1), F\left(C_{1}(1), \ldots, C_{n}(1)\right)\right)$ is the $\beta$ th ranked alternative and the top alternative respectively among $F\left(C_{1}(1), \ldots, C_{n}(1)\right)$ for agent 1 , the agent with the smallest index, at $C_{1}(1)$.
} 
each $t=\left(t_{1}, \ldots, t_{n}\right) \in \mathbb{N}^{n}$, and each $C=\left(C_{1}, \ldots, C_{n}\right) \in \tilde{\mathcal{C}}, g\left(\left(\alpha_{i}, t_{i}, C_{i}\right)_{i \in N}\right)=z\left(a_{\alpha}, t, C\right)$. Since $z$ satisfies (i)-(iv) in Definition $4,(M, g)$ satisfies the following four properties: (i') for each $\alpha$, each $t$, and each $C, g(\alpha, t, C) \in F\left(C_{1}\left(t_{1}\right), \ldots, C_{n}\left(t_{n}\right)\right)$, (ii') for each $\alpha$ and each $C$, $g(\alpha,(1, \ldots, 1), C)=a_{\alpha}$, (iii') for each $\alpha$, each $t$, and each $C, C^{\prime}$, if $C_{i}\left(\tau_{i}\right)=C_{i}^{\prime}\left(\tau_{i}\right)$ for each $i$ and each $\tau_{i} \leq t_{i}$, then $g(\alpha, t, C)=g\left(\alpha, t, C^{\prime}\right)$, and (iv') for each $i$, each $\alpha_{i}$, each $C_{i}$, and each $t_{i} \geq 2$, message $\left(\alpha_{i}, t_{i}, C_{i}\right)$ very weakly dominates another message $\left(\alpha_{i}, t_{i}-1, C_{i}\right)$ at $C_{i}\left(t_{i}\right)$ with respect to material preferences.

We show that the mechanism $(M, g)$ implements $F$ in undominated strategies under partial honesty, and then show that this mechanism is bounded.

STEP 2: We show that the mechanism $(M, g)$ implements all the alternatives in $F(P)$ for every $P \in \mathcal{D}$, i.e., $\{g(m) \mid m \in U(P)\} \supseteq F(P)$.

We can show that conditions (a) and (b) in Proposition 9 imply that for each $P_{i} \in \mathcal{D}_{i}$, messages of the form in (b) in Proposition 9 are undominated:

Claim 1. Suppose that the sincerity ordering satisfies conditions (a) and (b) in Proposition 9. Then, for each agent $i \in N$, each preference $P_{i} \in \mathcal{D}_{i}$, and each integer $\alpha_{i} \in\{1, \ldots,|A|\}$, message $m_{i}=\left(\alpha_{i}, 1,\left(P_{i}, P_{i}, \ldots\right)\right) \in M_{i}$ is undominated at $P_{i}$.

proof of Claim 1. Fix an agent $i \in N$, a preference $P_{i} \in \mathcal{D}_{i}$, and an integer $\alpha_{i} \in\{1, \ldots,|A|\}$ arbitrarily. Let $m_{i}=\left(\alpha_{i}, 1,\left(P_{i}, P_{i}, \ldots\right)\right)$. We show that for each $m_{i}^{\prime}=\left(\alpha_{i}^{\prime}, t_{i}^{\prime}, C_{i}^{\prime}\right) \neq m_{i}, m_{i}^{\prime}$ does not dominate $m_{i}$ at $P_{i}$ by considering two separate cases: (I) $C_{i}^{\prime} \neq\left(P_{i}, P_{i}, \ldots\right)$ and (II) $C_{i}^{\prime}=\left(P_{i}, P_{i}, \ldots\right)$.

Case (I): Fix $m_{i}^{\prime}=\left(\alpha_{i}^{\prime}, t_{i}^{\prime}, C_{i}^{\prime}\right) \in M_{i}$ such that $C_{i}^{\prime} \neq\left(P_{i}, P_{i}, \ldots\right)$ arbitrarily. By (ii') in Step 1, for each $P_{-i} \in \mathcal{D}_{-i}$ and each $a \in F(P)$, there exists $\alpha_{-i} \in\{1, \ldots,|A|\}^{n-1}$ such that $g\left(m_{i}, m_{-i}\right)=a$ where $m_{j}=\left(\alpha_{j}, 1,\left(P_{j}, P_{j}, \ldots\right)\right)$ for each $j \neq i$. Letting $a=$ $r^{1}\left(P_{i}, F(P)\right)$, there exists $m_{-i}$ such that $g\left(m_{i}, m_{-i}\right) R_{i} g\left(m_{i}^{\prime}, m_{-i}\right)$. By conditions (a) and (b) in Proposition 9, we have $\left(m_{i}, m_{-i}\right) \succ_{i}^{P_{i}}\left(m_{i}^{\prime}, m_{-i}\right)$ for this $m_{-i}$, and thus $m_{i}^{\prime}$ cannot dominate $m_{i}$ at $P_{i}$.

Case (II): Fix $m_{i}^{\prime}=\left(\alpha_{i}^{\prime}, t_{i}^{\prime},\left(P_{i}, P_{i}, \ldots\right)\right) \in M_{i}$ arbitrarily. If $\left|F\left(P_{i}, P_{-i}\right)\right|=1$ for every $P_{-i} \in \mathcal{D}_{-i}$, (i') in Step 1 implies that both $m_{i}$ and $m_{i}^{\prime}$ implement the same alternative uniquely assigned by $F$. In this case, $m_{i}^{\prime}$ does not dominate $m_{i}$ at $P_{i}$ by condition (a) in Proposition 9.

Suppose that $\left|F\left(P_{i}, P_{-i}\right)\right| \geq 2$ for some $P_{-i} \in \mathcal{D}_{-i}$. By condition (iv') in Step 1, $g\left(\tilde{m}_{i}, m_{-i}\right) R_{i} g\left(m_{i}^{\prime}, m_{-i}\right)$ for all $m_{-i} \in M_{-i}$ where $\tilde{m}_{i}=\left(\alpha_{i}^{\prime}, 1,\left(P_{i}, P_{i}, \ldots\right)\right) \in M_{i}$. By condition (a) in Proposition 9, $\tilde{m}_{i}$ very weakly dominates $m_{i}^{\prime}$ at $P_{i}$. If $\alpha_{i}^{\prime}=\alpha_{i}$, then $\tilde{m}_{i}=m_{i}$ and this is not dominated by $m_{i}^{\prime}$ at $P_{i}$. Suppose $\alpha_{i}^{\prime} \neq \alpha_{i}$. By (ii') in Step 1, there exists $\alpha_{-i} \in\{1, \ldots,|A|\}^{n-1}$ such that $g\left(m_{i}, m_{-i}\right)=r^{1}\left(P_{i}, F(P)\right)$ and $g\left(\tilde{m}_{i}, m_{-i}\right) \neq r^{1}\left(P_{i}, F(P)\right)$ 
where $m_{j}=\left(\alpha_{j}, 1,\left(P_{j}, P_{j}, \ldots\right)\right)$ for each $j \neq i$. Since $g\left(m_{i}, m_{-i}\right) P_{i} g\left(\tilde{m}_{i}, m_{-i}\right), \tilde{m}_{i}$ does not dominate $m_{i}$ at $P_{i}$. Since $m_{i}^{\prime}$ is very weakly dominated by $\tilde{m}_{i}$ at $P_{i}, m_{i}^{\prime}$ does not dominate $m_{i}$ at $P_{i}$. This completes the proof.

By (ii') in Step 1, for each $P \in \mathcal{D}$ and each $a \in F(P)$, there exists $\alpha \in\{1, \ldots,|A|\}^{n}$ such that $g\left(\left(\alpha_{i}, 1,\left(P_{i}, P_{i}, \ldots\right)\right)_{i \in N}\right)=a$. By Claim 1, the mechanism $(M, g)$ implements all alternatives in $F(P)$ in undominated strategies at every preference profile $P \in \mathcal{D}$, i.e., $\{g(m) \mid m \in U(P)\} \supseteq F(P)$.

Step 3: We show that $(M, g)$ implements no alternative in $A \backslash F(P)$ in undominated strategies for any $P \in \mathcal{D}$, i.e., $\{g(m) \mid m \in U(P)\} \subseteq F(P)$ for each $P \in \mathcal{D}$. To show this, the following claim is essential:

Claim 2. Suppose that the sincerity ordering satisfies condition (c) in Proposition 9. For each $i \in N$, each $m_{i}=\left(\alpha_{i}, t_{i}, C_{i}\right) \in M_{i}$, and each $P_{i} \in \mathcal{D}_{i}, m_{i}$ is very weakly dominated at $P_{i}$ by $\tilde{m}_{i}:=\left(\alpha_{i}, t_{i}+1, \tilde{C}_{i}\right) \in M_{i}$, where $\tilde{C}_{i} \in \tilde{\mathcal{C}}_{i}$ is a chain defined by $\tilde{C}_{i}\left(\tau_{i}\right)=C_{i}\left(\tau_{i}\right)$ if $\tau_{i} \leq t_{i}$, and $\tilde{C}_{i}\left(\tau_{i}\right)=P_{i}$ if $\tau_{i} \geq t_{i}+1$.

proof of Claim 2. Let $\tilde{m}_{i}^{\prime}=\left(\alpha_{i}, t_{i}, \tilde{C}_{i}\right) \in M_{i}$. By (iii') in Step 1, $g\left(\tilde{m}_{i}^{\prime}, m_{-i}\right)=g\left(m_{i}, m_{-i}\right)$ for all $m_{-i} \in M_{-i}$. Condition (iv') in Step 1 implies that $\tilde{m}_{i}$ very weakly dominates both $\tilde{m}_{i}^{\prime}$ and $m_{i}$ at $P_{i}$ with respect to material preferences. By condition (c) in Proposition 9, either $\tilde{m}_{i} S_{i}^{P_{i}} m_{i}$ or $\tilde{m}_{i} I\left(S_{i}^{P_{i}}\right) m_{i}$. Therefore, $\tilde{m}_{i}$ very weakly dominates $m_{i}$ at $P_{i}$.

Now, we show that for each $P \in \mathcal{D}$, any alternative in $A \backslash F(P)$ is not implemented in undominated strategies at $P$. Fix a preference profile $P \in \mathcal{D}$ arbitrarily. Suppose that an undominated message profile $m \in U(P)$ implements an alternative $a=g(m)$. For each $i \in N$, let $M_{i}^{P_{i}} \subset M_{i}$ be the set of messages $\left(\alpha_{i}, t_{i}, C_{i}\right)$ such that $C_{i}\left(\tau_{i}\right)=P_{i}$ for each $\tau_{i} \geq t_{i}$. By Claim 2, for each $i \in N$, there exists $\tilde{m}_{i} \in M_{i}^{P_{i}}$ such that $\tilde{m}_{i}$ very weakly dominates $m_{i}$ at $P_{i}$. Since $m_{i}$ is undominated at $P_{i}, \tilde{m}_{i}$ does not dominate $m_{i}$ at $P_{i}$. This implies that $g\left(\tilde{m}_{i}, m_{-i}\right)=g\left(m_{i}, m_{-i}\right)$ for all $m_{-i} \in M_{-i}$. Since this holds for all $i \in N$, $g(\tilde{m})=g(m)=a$. By (i') in Step 1, we have $g(\tilde{m}) \in F(P)$. Since this holds for all $m \in U(P),\{g(m) \mid m \in U(P)\} \subseteq F(P)$. Combined with Step 2, we have shown that $\{g(m) \mid m \in U(P)\}=F(P)$ for each $P \in \mathcal{D}$, i.e., $(M, g)$ implements $F$ in undominated strategies.

STEP 4: We prove boundedness of the mechanism $(M, g)$. Before showing boundedness, we show the following claim:

Claim 3. Suppose that $F$ satisfies strong chain dominance, and let $z: A \times \mathbb{N}^{n} \times \mathcal{C} \rightarrow A$ be the function given in Definition 4. Then, there exists a function $\tilde{z}: A \times \mathbb{N}^{n} \times \mathcal{C} \rightarrow A$ 
satisfying conditions (i)-(iv) in Definition 4, and (v) for each $i \in N$, each $K_{i} \in \mathbb{N}$, and each $C_{i} \in \tilde{\mathcal{C}}_{i}^{K_{i}}$, we have $\tilde{z}\left(a,\left(t_{i}, t_{-i}\right),\left(C_{i}, C_{-i}\right)\right)=\tilde{z}\left(a,\left(K_{i}, t_{-i}\right),\left(C_{i}, C_{-i}\right)\right)$ for each $t_{i} \geq K_{i}$, each $t_{-i} \in \mathbb{N}^{n-1}$, each $C_{-i} \in \mathcal{C}_{-i}$, and each $a \in F\left(C_{1}(1), \ldots, C_{n}(1)\right)$.

proof of Claim 3. Fix a chain profile $C \in \mathcal{C}$. For each $i \in N$, we define $K_{i}$ as follows: if $C_{i} \in \tilde{\mathcal{C}}_{i}$, let $K_{i}$ be an integer such that $C_{i} \in \tilde{\mathcal{C}}_{i}^{K_{i}}$. If $C_{i} \notin \tilde{\mathcal{C}}_{i}$, let $K_{i}=\infty$ for convenience. For each $t \in \mathbb{N}^{n}$ and each $a \in F\left(C_{1}(1), \ldots, C_{n}(1)\right)$, let $\tilde{z}(a, t, C)=z\left(a,\left(\min \left\{K_{i}, t_{i}\right\}\right)_{i \in N}, C\right)$. Since $z$ satisfies conditions (i)-(iii) in Definition $4, \tilde{z}$ also satisfies these conditions. For each $i \in N, \tilde{z}$ satisfies condition (iv) in Definition 4 because if $t_{i} \leq K_{i}$, then it follows from the fact that $z$ satisfies condition (iv), and if $t_{i} \geq K_{i}+1$, then $\tilde{z}\left(a,\left(t_{i}-1, t_{-i}\right), C\right)=\tilde{z}(a, t, C)$ by the definition of $\tilde{z}$. This equality also implies $(\mathrm{v})$ in the statement.

By Claim 3, we can assume without loss of generality that for each $i \in N$ and each $m_{i} \in\left(\alpha_{i}, t_{i}, C_{i}\right) \in M_{i}$, if $C_{i} \in \tilde{\mathcal{C}}_{i}^{K_{i}}$ for some $K_{i} \leq t_{i}$, then $g\left(m_{i}, m_{-i}\right)=g\left(\left(\alpha_{i}, K_{i}, C_{i}\right), m_{-i}\right)$ for all $m_{-i} \in M_{-i}$. By (a) in Proposition 9, $\left(\alpha_{i}, t_{i}, C_{i}\right)$ and $\left(\alpha_{i}, K_{i}, C_{i}\right)$ are equally sincere. Thus, for each $i \in N$ and each $m_{i} \in\left(\alpha_{i}, t_{i}, C_{i}\right) \in M_{i}$, if $C_{i} \in \tilde{\mathcal{C}}_{i}^{K_{i}}$ for some $K_{i} \in \mathbb{N}$, then we can assume $t_{i} \leq K_{i}$ without loss of generality. Under this assumption, for each $i \in N$ and each $\tilde{C}_{i} \in \tilde{\mathcal{C}}_{i}^{K_{i}}$, we can focus on a finite set of messages $\left\{\left(\alpha_{i}, t_{i}, C_{i}\right) \in M_{i} \mid \alpha_{i} \in\right.$ $\left.\{1, \ldots,|A|\}, t_{i} \leq K_{i}, C_{i}=\tilde{C}_{i}\right\}$.

We complete the proof by proving boundedness of the mechanism.

Claim 4. Suppose that the sincerity ordering satisfies conditions (a), (c), and (d) in Proposition 9. Then, the mechanism $(M, g)$ is bounded.

proof of Claim 4. A necessary and sufficient condition of boundedness is that for each $i \in N$, each $P_{i} \in \mathcal{D}_{i}$, and each $m_{i} \in M_{i}$, there exists $\tilde{m}_{i} \in U_{i}\left(P_{i}\right)$ which very weakly dominates $m_{i}$ at $P_{i}$. By Claim 2, it suffices to show this for each $m_{i} \in M_{i}^{P_{i}} \subset M_{i}$. Let $\hat{M}_{i}^{P_{i}} \subseteq M_{i}^{P_{i}}$ be the set of messages $m_{i} \in M_{i}^{P_{i}}$ such that $m_{i}$ is not very weakly dominated by any undominated message $\tilde{m}_{i} \in U_{i}\left(P_{i}\right)$. We want to show that $\hat{M}_{i}^{P_{i}}$ is empty for each $i \in N$ and each $P_{i} \in \mathcal{D}_{i}$.

Assume on the contrary that $\hat{M}_{i}^{P_{i}}$ is nonempty for some $i \in N$ and $P_{i} \in \mathcal{D}_{i}$. We fix such $i \in N$ and $P_{i} \in \mathcal{D}_{i}$. First, we show that for each $m_{i}=\left(\alpha_{i}, t_{i}, C_{i}\right) \in \hat{M}_{i}^{P_{i}}$, there exists $\tilde{m}_{i} \in M_{i}^{P_{i}}$ such that $g\left(\tilde{m}_{i}, m_{-i}\right) P_{i} g\left(m_{i}, m_{-i}\right)$ for all $m_{-i} \in M_{-i}$. Fix a message $m_{i}=\left(\alpha_{i}, t_{i}, C_{i}\right) \in \hat{M}_{i}^{P_{i}}$ arbitrarily. By condition (d) in Proposition 9, there exist only finite messages $\hat{m}_{i}=\left(\alpha_{i}, t_{i}, \hat{C}_{i}\right) \in M_{i}$ such that $\hat{C}_{i} \in \tilde{\mathcal{C}}_{i}$, and $\hat{m}_{i}$ is not less sincere than $m_{i}$ at $P_{i}$. Let $s_{i}<\infty$ be the number of such messages. Let $K_{i}$ be the integer such that $C_{i} \in \tilde{\mathcal{C}}_{i}^{K_{i}}$. By Claim 3, we can focus on the finite set of messages $\left\{\left(\tilde{\alpha}_{i}, \tilde{t}_{i}, C_{i}\right) \in M_{i} \mid \tilde{\alpha}_{i} \in\{1, \ldots,|A|\}, \tilde{t}_{i} \leq\right.$ $\left.K_{i}\right\}$. In this set, the number of messages which are not less sincere than $m_{i}$ at $P_{i}$ is $|A| K_{i} s_{i}$ $(<\infty)$ by condition (a) in Proposition 9 . Since $m_{i}$ is not very weakly dominated by any 
undominated message at $P_{i}, m_{i} \notin U_{i}\left(P_{i}\right)$ (because $m_{i}$ very weakly dominates itself in a trivial sense). Since $m_{i}$ is dominated at $P_{i}$ but is not dominated by any undominated message at $P_{i}$, there must exist infinitely many messages that dominate $m_{i}$. Since there exist only finite messages which are not less sincere than $m_{i}$ at $P_{i}$, there exists $m_{i}^{\prime} \in M_{i}$ which dominates $m_{i}$ at $P_{i}$ and satisfies $m_{i} S_{i}^{P_{i}} m_{i}^{\prime}$. By the definition of partial honesty, such a message $m_{i}^{\prime}$ satisfies $g\left(m_{i}^{\prime}, m_{-i}\right) P_{i} g\left(m_{i}, m_{-i}\right)$ for all $m_{-i} \in M_{-i}$. By Claim 2, there exists $\tilde{m}_{i} \in M_{i}^{P_{i}}$ such that $g\left(\tilde{m}_{i}, m_{-i}\right) P_{i} g\left(m_{i}, m_{-i}\right)$ for all $m_{-i} \in M_{-i}$. Thus, for each $m_{i} \in \hat{M}_{i}^{P_{i}}$, we can find $d_{i}^{P_{i}}\left(m_{i}\right) \in M_{i}^{P_{i}}$ such that $g\left(d_{i}^{P_{i}}\left(m_{i}\right), m_{-i}\right) P_{i} g\left(m_{i}, m_{-i}\right)$ for all $m_{-i} \in M_{-i}$.

Next, we derive a contradiction. If there exists an undominated message $\bar{m}_{i} \in U_{i}\left(P_{i}\right)$ which very weakly dominates $d_{i}^{P_{i}}\left(m_{i}\right)$ at $P_{i}$, then $\bar{m}_{i}$ also very weakly dominates $m_{i}$ at $P_{i}$, contradicting the assumption $m_{i} \in \hat{M}_{i}^{P_{i}}$. Thus, we have $d_{i}^{P_{i}}\left(m_{i}\right) \in \hat{M}_{i}^{P_{i}}$ for each $m_{i} \in \hat{M}_{i}^{P_{i}}$. For a fixed $m_{i}^{0} \in \hat{M}_{i}^{P_{i}}$, we can define an infinite sequence of messages $m_{i}^{0}, m_{i}^{1}, m_{i}^{2}, \ldots$ by $m_{i}^{k}=d_{i}^{P_{i}}\left(m_{i}^{k-1}\right)$ for each $k \geq 1$. By the definition of $d_{i}^{P_{i}}$, for each $k \geq 1, g\left(m_{i}^{k}, m_{-i}\right) P_{i}$ $g\left(m_{i}^{k-1}, m_{-i}\right)$ for all $m_{-i} \in M_{-i}$. This is impossible because the set of alternatives is finite. Hence, $\hat{M}_{i}^{P_{i}}$ must be empty for each $i \in N$ and each $P_{i} \in \mathcal{D}_{i}$.

\section{A.2 Proof of Proposition 2}

\section{A.2.1 Proof of necessity}

We show the "only if" part.

Suppose that a finite mechanism $(M, g)$ implements $F$ in undominated strategies. Since any finite mechanism is bounded, we can apply the arguments in Appendix A.1.1. For each agent $i \in N$, each $\bar{P} \in \mathcal{D}$, each $a \in A$, and and each chain $C_{i} \in \mathcal{C}_{i}$ with $C_{i}(1)=\bar{P}_{i}$, let $\left(m_{i}^{t_{i}}\left(a, \bar{P}, C_{i}\right)\right)_{t_{i}=1}^{\infty}$ be the sequence defined in Appendix A.1.1. We can define $z(a, t, C):=$ $g\left(m_{1}^{t_{1}}\left(a, \bar{P}, C_{i}\right), \ldots, m_{n}^{t_{n}}\left(a, \bar{P}, C_{i}\right)\right)$ for each $t \in \mathbb{N}^{n}$, where $\bar{P}=\left(C_{1}(1), \ldots, C_{n}(1)\right)$. By the arguments in Appendix A.1.1, $z$ satisfies conditions (i)-(iv) in Definition 4, i.e., $F$ satisfies strong chain dominance.

Fix an agent $i \in N$ and a chain $C_{i} \in \mathcal{C}_{i}$, arbitrarily. For each $t_{i} \in \mathbb{N}$, let $m_{i}^{t_{i}}\left(\cdot,\left(C_{i}(1), \cdot\right), C_{i}\right): A \times \mathcal{D}_{-i} \rightarrow M_{i}$ be the function which assigns $m_{i}^{t_{i}}\left(a,\left(C_{i}(1), \bar{P}_{-i}\right), C_{i}\right)$ from given $a \in A$ and $\bar{P}_{-i} \in \mathcal{D}_{-i}$. By the definition of the function $z$,

$$
\begin{aligned}
\left|\left\{\left.z\right|_{i, t_{i}, C_{i}} ; t_{i} \in \mathbb{N}\right\}\right| & =\left|\left\{m_{i}^{t_{i}}\left(\cdot,\left(C_{i}(1), \cdot\right), C_{i}\right) ; t_{i} \in \mathbb{N}\right\}\right| \\
& \leq\left|M_{i}\right|^{|A| \cdot\left|\mathcal{D}_{-i}\right|} .
\end{aligned}
$$

We complete the proof by letting $L=\max _{i \in N}\left|M_{i}\right|^{|A| \cdot\left|\mathcal{D}_{-i}\right|}$. 


\section{A.2.2 Proof of sufficiency}

We show the "if" part.

Let $z$ be the function given in the statement. For each $i \in N$ and each $C_{i} \in \mathcal{C}_{i}$, let $\bar{t}\left(C_{i}\right)=\min \left\{t_{i} \mid C_{i}\left(t_{i}^{\prime}\right)=C_{i}\left(t_{i}\right)\right.$ for $\left.\forall t_{i}^{\prime} \geq t_{i}\right\}$ if there exists such $t_{i}$, and $\bar{t}\left(C_{i}\right)=\infty$ if there exists no such $t_{i}$. Let $\tilde{z}$ be the function defined by, for each $a \in A$, each $t \in \mathbb{N}^{n}$, and each $C \in \mathcal{C}$,

$$
\tilde{z}(a, t, C)=z\left(a,\left(\min \left\{t_{i}, \bar{t}\left(C_{i}\right)\right\}\right)_{i \in N}, C\right) .
$$

By this definition, if $C_{i}\left(t_{i}^{\prime}\right)=C_{i}\left(t_{i}\right)$ for all $t_{i}^{\prime} \geq t_{i}$, then $\left.\tilde{z}\right|_{i, t_{i}^{\prime}, C_{i}}=\left.\tilde{z}\right|_{i, t_{i}, C_{i}}$ for all $t_{i}^{\prime} \geq t_{i}$. Since it is assumed that $\left|\left\{\left.z\right|_{i, t_{i}, C_{i}} ; t_{i} \in \mathbb{N}\right\}\right| \leq L$ for each $i \in N$ and each $C_{i} \in \mathcal{C}_{i}$, we also have $\left|\left\{\left.\tilde{z}\right|_{i, t_{i}, C_{i}} ; t_{i} \in \mathbb{N}\right\}\right| \leq L$. Since $z$ satisfies conditions (i)-(iv) in Definition $4, \tilde{z}$ satisfies these conditions.

For each $i \in N$, each $t_{i} \in \mathbb{N}$, and each $C_{i} \in \mathcal{C}_{i}$, let

$$
\tau_{i}^{C_{i}}\left(t_{i}\right)=\min \left\{t_{i}^{\prime} \in \mathbb{N}\left|C_{i}\left(t_{i}^{\prime}\right)=C_{i}\left(t_{i}\right), \tilde{z}\right|_{i, t_{i}^{\prime}, C_{i}}=\left.\tilde{z}\right|_{i, t_{i}, C_{i}}\right\}
$$

Let $\bar{L}:=L \cdot \max _{i \in N}\left|\mathcal{D}_{i}\right|$. Since $\left|\left\{\left.\tilde{z}\right|_{i, t_{i}, C_{i}} ; t_{i} \in \mathbb{N}\right\}\right| \leq L$ for each $i \in N$ and each $C_{i} \in \mathcal{C}_{i}$, we have $\tau_{i}^{C_{i}}\left(t_{i}\right)<t_{i}$ for each $i \in N$, each $t_{i} \geq \bar{L}+1$, and each $C_{i} \in \mathcal{C}_{i}$.

Let us consider the mechanism $\Gamma=(M, g)$ defined in Step 1 in Appendix A.1.2 with respect to the function $\tilde{z}$ under sincerity ordering $(*)$. For each $i \in N$, let $\mathcal{C}_{i}^{*}$ be the set of chains $C_{i} \in \tilde{\mathcal{C}}_{i}$ such that there exists $\bar{t}_{i} \in \mathbb{N}$ such that $\tau_{i}^{C_{i}}\left(t_{i}\right)=t_{i}$ for each $t_{i} \leq \bar{t}_{i}$, and $C_{i}\left(t_{i}\right)=C_{i}\left(\bar{t}_{i}\right)$ for each $t_{i} \geq \bar{t}_{i}$. Since $\tau_{i}^{C^{C}}\left(t_{i}\right)=t_{i}$ implies $t_{i} \leq \bar{L}$, every $C_{i} \in \mathcal{C}_{i}^{*}$ satisfies $C_{i}\left(t_{i}\right)=C_{i}(\bar{L})$ for each $t_{i} \geq \bar{L}$. Let $M_{i}^{*}=\{1, \ldots,|A|\} \times\{1, \ldots, \bar{L}\} \times \mathcal{C}_{i}^{*}, \mathcal{C}^{*}=\prod_{i \in N} \mathcal{C}_{i}^{*}$, and $M^{*}=\prod_{i \in N} M_{i}^{*}$. We show that the restricted mechanism $\Gamma^{*}=\left(M^{*},\left.g\right|_{M^{*}}\right)$ also implements $F$ in undominated strategies. The same argument in the proof of Claim 1 in Appendix A.1.2 shows that $\left\{g(m) \mid m \in U\left(P, \Gamma^{*}\right)\right\} \supseteq F(P)$ for every $P \in \mathcal{D}$ because $\left(P_{i}, P_{i}, \ldots\right) \in \mathcal{C}_{i}^{*}$ for each $i \in N$ and $P_{i} \in \mathcal{D}_{i}$.

We show that $\left\{g(m) \mid m \in U\left(P, \Gamma^{*}\right)\right\} \subseteq F(P)$ for every $P \in \mathcal{D}$. Note that if a message $m_{i}$ very weakly dominates another message $m_{i}^{\prime}$ in mechanism $(M, g)$, then $m_{i}$ very weakly dominates $m_{i}^{\prime}$ in the restricted mechanism $\left(M^{*},\left.g\right|_{M^{*}}\right)$. In the following proof, we mean dominance in $(M, g)$ by "very weakly dominates". Fix $i \in N, m_{i}=\left(\alpha_{i}, t_{i}, C_{i}\right) \in M_{i}^{*}$, and $P_{i}^{\prime} \in \mathcal{D}_{i} \backslash\left\{C_{i}\left(t_{i}\right)\right\}$, arbitrarily. By (i') in Step 1 in Appendix A.1.2, it suffices to show that there exists $m_{i}^{*}=\left(\alpha_{i}, t_{i}^{*}, C_{i}^{*}\right) \in M_{i}^{*}$ which very weakly dominates $m_{i}$ at $P_{i}^{\prime}$, and satisfies $C_{i}^{*}\left(t_{i}^{*}\right)=P_{i}^{\prime}$. Let $\tilde{m}_{i}=\left(\alpha_{i}, t_{i}+1, \tilde{C}_{i}\right) \in M_{i}$ where $\tilde{C}_{i} \in \mathcal{C}_{i}$ is such that $\tilde{C}_{i}\left(k_{i}\right)=C_{i}\left(k_{i}\right)$ for each $k_{i} \leq t_{i}$, and $\tilde{C}_{i}\left(k_{i}\right)=P_{i}^{\prime}$ for each $k_{i} \geq t_{i}+1$. By Claim 2 in Appendix A.1.2, $\tilde{m}_{i}$ very 
weakly dominates $m_{i}$ at $P_{i}^{\prime}$. Let $m_{i}^{*}=\left(\alpha_{i}, t_{i}^{*}, C_{i}^{*}\right)$ where $t_{i}^{*}=\tau_{i}^{\tilde{C}_{i}}\left(t_{i}+1\right)$, and $C_{i}^{*}$ is such that for each $k_{i} \in \mathbb{N}$,

$$
C_{i}^{*}\left(k_{i}\right)= \begin{cases}\tilde{C}_{i}\left(k_{i}\right) & \text { if } t_{i} \leq \tau_{i}^{\tilde{C}_{i}}\left(t_{i}+1\right), \\ P_{i}^{\prime} & \text { if } t_{i} \geq \tau_{i}^{\tilde{C}_{i}}\left(t_{i}+1\right)+1\end{cases}
$$

Since $C_{i}^{*}\left(\tau_{i}^{\tilde{C}_{i}}\left(t_{i}+1\right)\right)=\tilde{C}_{i}\left(t_{i}+1\right)=P_{i}^{\prime}, C_{i}^{*}$ is the chain given by removing the subsequence between $\tilde{C}_{i}\left(\tau_{i}^{\tilde{C}_{i}}\left(t_{i}+1\right)\right)$ and $\tilde{C}_{i}\left(t_{i}\right)$. By the definition of $\tau_{i}^{\tilde{C}_{i}}, C_{i}^{*} \in \mathcal{C}_{i}^{*}, C_{i}^{*}\left(t_{i}^{*}\right)=P_{i}^{\prime}$ and $\left.\tilde{z}\right|_{i, t_{i}^{*}, C_{i}^{*}}=\left.\tilde{z}\right|_{i, t_{i}+1, \tilde{C}_{i}}$, which implies $g\left(m_{i}^{*}, m_{-i}\right)=g\left(\tilde{m}_{i}, m_{-i}\right)$ for all $m_{-i} \in M_{-i}$. By (c) in Proposition $9, m_{i}^{*}$ is not less sincere than $\left(\alpha_{i}, t_{i}^{*}, \tilde{C}\right)$, which is equally sincere to $\tilde{m}_{i}$ by (a) in Proposition 9. Since $\tilde{m}_{i}$ very weakly dominates $m_{i}$ at $P_{i}^{\prime}, m_{i}^{*}$ also very weakly dominates $m_{i}$ at $P_{i}^{\prime}$. Since $t_{i} \leq \bar{L}$, we have $\tau_{i}^{\tilde{C}_{i}}\left(t_{i}+1\right) \leq \bar{L}+1$. Assume that the equality holds, which is equivalent to $t_{i}=\bar{L}$ and $\tau_{i}^{\tilde{C}_{i}}(\bar{L}+1)=\bar{L}+1$. This implies that for each $t_{i}^{\prime} \leq \bar{L}$, $C_{i}\left(t_{i}^{\prime}\right) \neq C_{i}(\bar{L}+1)$ or $\left.\tilde{z}\right|_{i, t_{i}^{\prime}, C_{i}} \neq\left.\tilde{z}\right|_{i, \bar{L}_{i}, C_{i}}$. Since $C_{i} \in \mathcal{C}_{i}^{*}$ and $C_{i}\left(t_{i}\right)=C_{i}(\bar{L}) \neq P_{i}^{\prime}$, we have $C_{i}\left(t_{i}^{\prime}\right) \neq C_{i}\left(t_{i}^{\prime \prime}\right)$ or $\left.\tilde{z}\right|_{i, t_{i}^{\prime}, C_{i}} \neq\left.\tilde{z}\right|_{i, t_{i}^{\prime \prime}, C_{i}}$ for each $t_{i}^{\prime}<t_{i}^{\prime \prime} \leq \bar{L}+1$. This is impossible because $\left|\left\{\left.\tilde{z}\right|_{i, t_{i}^{\prime}, C_{i}} ; t_{i}^{\prime} \in \mathbb{N}\right\}\right| \leq L$. Therefore, $t_{i}^{*}=\tau_{i}^{\tilde{C}_{i}}\left(t_{i}+1\right) \leq \bar{L}$. Since $C_{i}^{*} \in \mathcal{C}_{i}^{*}$, we have $m_{i}^{*} \in M_{i}^{*}$. Therefore, for each $i \in N$, each $m_{i}=\left(\alpha_{i}, t_{i}, C_{i}\right) \in M_{i}^{*}$, and each $P_{i}^{\prime} \in \mathcal{D}_{i} \backslash\left\{C_{i}\left(t_{i}\right)\right\}$, there exists $m_{i}^{*} \in M_{i}^{*}$ which very weakly dominates $m_{i}$ at $P_{i}^{\prime}$. Hence, $\left(M^{*},\left.g\right|_{M^{*}}\right)$ implements $F$ in undominated strategies.

For each $i \in N$, let $\bar{M}_{i}^{*}=\left\{\left(\alpha_{i}, t_{i}, P_{i}^{1}, P_{i}^{2}, \ldots, P_{i}^{2 \bar{L}}\right) \mid \exists C_{i}^{*} \in \mathcal{C}_{i}^{*}\right.$ s.t. $C_{i}^{*}\left(k_{i}\right)=P_{i}^{k_{i}}$ for $\forall k_{i} \leq$ $2 \bar{L}\} \subset\{1, \ldots,|A|\} \times\{1, \ldots, \bar{L}\} \times\left(\mathcal{D}_{i}\right)^{2 \bar{L}}$ be the message space. Note that $P_{i}^{k_{i}}=P_{i}^{\bar{L}}$ for each $k_{i}$ with $\bar{L} \leq k_{i} \leq 2 \bar{L}$ because any $C_{i}^{*} \in \mathcal{C}_{i}^{*}$ satisfies $C_{i}^{*}\left(k_{i}\right)=C_{i}^{*}(\bar{L})$ for each $k_{i} \geq \bar{L}$. In this space, it is easy to see that $\left(\alpha_{i}, t_{i}, P_{i}^{1}, P_{i}^{2}, \ldots, P_{i}^{\bar{L}-1}, P_{i}^{\bar{L}}, P_{i}^{\bar{L}}, \ldots, P_{i}^{\bar{L}}\right) \in \bar{M}^{*}$ is more sincere than $\left(\bar{\alpha}_{i}, \bar{t}_{i}, \bar{P}_{i}^{1}, \bar{P}_{i}^{2}, \ldots, \bar{P}_{i}^{\bar{L}-1}, \bar{P}_{i}^{\bar{L}}, \bar{P}_{i}^{\bar{L}}, \ldots, \bar{P}_{i}^{\bar{L}}\right) \in \bar{M}^{*}$ with respect to the sincerity ordering (**) if and only if $\left(\alpha_{i}, t_{i}, P_{i}^{1}, P_{i}^{2}, \ldots, P_{i}^{\bar{L}-1}, P_{i}^{\bar{L}}, P_{i}^{\bar{L}}, P_{i}^{\bar{L}}, \ldots\right) \in M_{i}^{*}$ is more sincere than $\left(\bar{\alpha}_{i}, \bar{t}_{i}, \bar{P}_{i}^{1}, \bar{P}_{i}^{2}, \ldots, \bar{P}_{i}^{\bar{L}-1}, \bar{P}_{i}^{\bar{L}}, \bar{P}_{i}^{\bar{L}}, \bar{P}_{i}^{\bar{L}}, \ldots\right) \in M_{i}^{*}$ with respect to the sincerity ordering $\left(^{*}\right)$. The assignment function $\bar{g}^{*}: \bar{M}^{*} \rightarrow A$ is defined by $g\left(\left(\alpha_{i}, t_{i}, P_{i}^{1}, P_{i}^{2}, \ldots, P_{i}^{2 \bar{L}}\right)_{i \in N}\right)=$ $g\left(\left(\alpha_{i}, t_{i}, P_{i}^{1}, P_{i}^{2}, \ldots, P_{i}^{2 \bar{L}}, P_{i}^{2 \bar{L}}, P_{i}^{2 \bar{L}}, \ldots\right)_{i \in N}\right)$. The mechanism $\left(\bar{M}^{*}, \bar{g}^{*}\right)$ implements $F$ in undominated strategies because $\left(M^{*},\left.g\right|_{M^{*}}\right)$ implements $F$ in undominated strategies.

\section{A.3 Proof of Corollary 6}

First, let $F$ be the plurality correspondence. We distinguish three cases.

Case 1: $n=3$ and $|A|>2$. Let $a, b, c \in A$ and for each $i \in N$, let $P_{i}^{1}, P_{i}^{2} \in \mathcal{D}_{i}$ be such that:

$c P_{1}^{1} a P_{1}^{1} b$ and for each $d \in A \backslash\{a, b, c\}$, we have $b P_{1}^{1} d$, 
$a P_{1}^{2} c P_{1}^{2} b$ and for each $d \in A \backslash\{a, b, c\}$, we have $b P_{1}^{2} d$, $a P_{2}^{1} b P_{2}^{1} c$ and for each $d \in A \backslash\{a, b, c\}$, we have $c P_{2}^{1} d$, $b P_{2}^{2} a P_{2}^{2} c$ and for each $d \in A \backslash\{a, b, c\}$, we have $c P_{2}^{2} d$. $b P_{3}^{1} c P_{3}^{1} a$ and for each $d \in A \backslash\{a, b, c\}$, we have $a P_{3}^{1} d$, $c P_{3}^{2} b P_{3}^{2} a$ and for each $d \in A \backslash\{a, b, c\}$, we have $a P_{3}^{2} d$.

Let $z$ of $F$ be a function satisfying Conditions (i) to (iii) of Definition 4. Also, let $C \in \mathcal{C}$ be such that for each $i \in N$, we have $C_{i}=\left(P_{i}^{1}, P_{i}^{2}, \ldots\right)$ and $a_{1} \in F\left(\left(P_{i}^{1}\right)_{i \in N}\right)$, $z_{122}=z\left(a_{1},(1,2,2), C\right), z_{212}=z\left(a_{1},(2,1,2), C\right), z_{221}=z\left(a_{1},(2,2,1), C\right)$, and $z_{222}=$ $z\left(a_{1},(2,2,2), C\right)$.

By condition (i), $z_{122} \in F\left(P_{1}^{1}, P_{2}^{2}, P_{3}^{2}\right), z_{212} \in F\left(P_{1}^{2}, P_{2}^{1}, P_{3}^{2}\right), z_{221} \in F\left(P_{1}^{2}, P_{2}^{2}, P_{3}^{1}\right)$, and $z_{222} \in F\left(P_{1}^{2}, P_{2}^{2}, P_{3}^{2}\right)$. Suppose $z_{222} R_{1}^{2} z_{122}$ and $z_{222} R_{2}^{2} z_{212}$. Since $F\left(P_{1}^{1}, P_{2}^{2}, P_{3}^{2}\right)=\{c\}$, hence $z_{122}=c$, and $z_{222} R_{1}^{2} z_{122}$, we have $z_{222} \neq b$. Since $F\left(P_{1}^{2}, P_{2}^{1}, P_{3}^{2}\right)=\{a\}$, hence $z_{212}=a$, and $z_{222} R_{2}^{2} z_{212}$, we have $z_{222} \neq c$. Thus, $z_{222}=a$. Since $F\left(P_{1}^{2}, P_{2}^{2}, P_{3}^{1}\right)=\{b\}$, hence $z_{221}=b$, we have $z_{221} P_{3}^{2} z_{222}$, contradicting condition (iv) of Definition 4 , hence $F$ violates strong chain dominance.

Case 2: $n=5$ and $|A|=3$. Let $a, b, c \in A$ and for each $i \in\{1,2,3\}$, let $P_{i}^{1}, P_{i}^{2} \in \mathcal{D}_{i}$ be such that:

$c P_{1}^{1} a P_{1}^{1} b$,
$b P_{1}^{2} c P_{1}^{2} a$,
$b P_{2}^{1} c P_{2}^{1} a$,
$a P_{2}^{2} b P_{2}^{2} c$,

for $t=1,2$, we have $P_{3}^{t}=P_{2}^{t}$,

and let $P_{4}^{1}=P_{5}^{1}=P_{1}^{1}$.

Let $z$ of $F$ be a function satisfying Conditions (i) to (iii) of Definition 4. Also, let $C \in \mathcal{C}$ be such that for each $i \in\{1,2,3\}$, we have $C_{i}=\left(P_{i}^{1}, P_{i}^{2}, \ldots\right)$, for each $i \in\{4,5\}$, we have $C_{i}=$ $\left(P_{i}^{1}, \ldots\right)$, and $a_{1} \in F\left(\left(P_{i}^{1}\right)_{i \in N}\right), z_{211}=z\left(a_{1},(2,1,1,1,1), C\right), z_{221}=z\left(a_{1},(2,2,1,1,1), C\right)$, $z_{122}=z\left(a_{1},(1,2,2,1,1), C\right)$, and $z_{222}=z\left(a_{1},(2,2,2,1,1), C\right)$.

By condition (i), $z_{211} \in F\left(P_{1}^{2},\left(P_{i}^{1}\right)_{i \in N \backslash\{1\}}\right), z_{221} \in F\left(P_{1}^{2}, P_{2}^{2},\left(P_{i}^{1}\right)_{i \in N \backslash\{1,2\}}\right), z_{122} \in$ $F\left(P_{2}^{2}, P_{3}^{2},\left(P_{i}^{1}\right)_{i \in N \backslash\{2,3\}}\right)$, and $z_{222} \in F\left(P_{1}^{2}, P_{2}^{2}, P_{3}^{2}, P_{4}^{1}, P_{5}^{1}\right)$. Since $F\left(P_{2}^{2}, P_{3}^{2},\left(P_{i}^{1}\right)_{i \in N \backslash\{2,3\}}\right)=$ $\{c\}$, hence $z_{122}=c$, and $F\left(P_{1}^{2}, P_{2}^{2}, P_{3}^{2}, P_{4}^{1}, P_{5}^{1}\right)=\{a, c\}$, hence $z_{222}=a, c$. If $z_{222} R_{1}^{2} z_{122}$, $z_{222}=c$. Since $F\left(P_{1}^{2}, P_{2}^{2},\left(P_{i}^{1}\right)_{i \in N \backslash\{1,2\}}\right)=\{b, c\}$, hence $z_{221}=b, c$. If $z_{222} R_{3}^{2} z_{221}$, then $z_{221}=c$. Since $F\left(P_{1}^{2},\left(P_{i}^{1}\right)_{i \in N \backslash\{1\}}\right)=\{b\}$, hence $z_{211}=b$, we have $z_{211} P_{2}^{2} z_{221}$, contradicting condition (iv) of Definition 4 , hence $F$ violates strong chain dominance.

Case 3: $(n=4$ and $|A|>2),(n \geq 6$ and $|A|>2)$, or $(n=5$ and $|A|>3)$. By Ohseto (1994), $F$ violates strategy-resistance, hence it violates strong chain dominance.

Second, let $F$ be a positional scoring rule with $s_{1}>s_{2}>0$. We also distinguish three 
cases.

Case $1^{\prime}:$ Suppose $n=2$ and $|A|>2$. Let $a, b, c \in A$ and for each $i \in N$, let $P_{i}^{1}, P_{i}^{2} \in \mathcal{D}_{i}$ be such that:

$a P_{1}^{1} c P_{1}^{1} b$ and for each $d \in A \backslash\{a, b, c\}$, we have $b P_{1}^{1} d$,

$a P_{1}^{2} b P_{1}^{2} c$ and for each $d \in A \backslash\{a, b, c\}$, we have $c P_{1}^{2} d$,

$b P_{2}^{1} c P_{2}^{1} a$ and for each $d \in A \backslash\{a, b, c\}$, we have $a P_{2}^{1} d$,

$b P_{2}^{2} a P_{2}^{2} c$ and for each $d \in A \backslash\{a, b, c\}$, we have $c P_{2}^{2} d$.

Let $z$ of $F$ be a function satisfying conditions (i) to (iii) of Definition 4. Also, let $C \in \mathcal{C}$ be such that for each $i \in N$, we have $C_{i}=\left(P_{i}^{1}, P_{i}^{2}, \ldots\right)$, and $a_{1} \in F\left(P_{1}^{1}, P_{2}^{1}\right), z_{12}=$ $z\left(a_{1},(1,2), C\right), z_{21}=z\left(a_{1},(2,1), C\right), z_{22}=z\left(a_{1},(2,2), C\right)$. By condition $(\mathrm{i}), z_{21} \in F\left(P_{1}^{2}, P_{2}^{1}\right)$, $z_{12} \in F\left(P_{1}^{1}, P_{2}^{2}\right)$, and $z_{22} \in F\left(P_{1}^{2}, P_{2}^{2}\right)$. As $s_{2}>0$, we have $F\left(P_{1}^{1}, P_{2}^{2}\right)=\{a\}$, hence $z_{12}=a$, and $F\left(P_{1}^{2}, P_{2}^{1}\right)=\{b\}$, hence $z_{21}=b$. If $z_{22} R_{1}^{2} z_{12}$, then $z_{22}=a$. Thus, hence $z_{21} P_{2}^{2} z_{22}$, contradicting condition (iv) of Definition 4 , hence $F$ violates strong chain dominance.

Case $2^{\prime}$ : Suppose $n=3$ and $|A|>2$. Let $a, b, c \in A$ and for each $i \in N$, let $P_{i}^{1}, P_{i}^{2} \in \mathcal{D}_{i}$ be as in Case 1. Then, $F$ coincides with the plurality correspondence, hence $F$ violates strong chain dominance.

Case $3^{\prime}$ : Suppose $n \geq 4$ and $|A|>2$. Let $S, S^{\prime} \subset N$ such that $1,2 \notin S \cup S^{\prime}, S \cap S^{\prime}=\emptyset$, and $|S|=(n / 2)-1$. Let $a, b, c \in A$ and $P_{1}^{1}, P_{1}^{2} \in \mathcal{D}_{1}$ be such that:

$b P_{1}^{1} c P_{1}^{1} a$ and for each $d \in A \backslash\{a, b, c\}$, we have $c P_{1}^{1} d$,

$b P_{1}^{2} a P_{1}^{2} c$ and for each $d \in A \backslash\{a, b, c\}$, we have $a P_{1}^{2} d$.

For each $i \in S \cup S^{\prime}$, we have $P_{i}^{1} \in \mathcal{D}_{i}$ such that:

if $i \in S$, then $a P_{i}^{1} b P_{i}^{1} c$, if $i \in S^{\prime}$, then $b P_{i}^{1} a P_{i}^{1} c$, and for each $d \in A \backslash\{a, b, c\}$, we have $c P_{i}^{1} d$.

If $n$ is even, then suppose $\left|S^{\prime}\right|=(n / 2)-1$ and let $P_{2}^{1} \in \mathcal{D}_{2}$ be such that:

$a P_{2}^{1} c P_{2}^{1} b$ and for each $d \in A \backslash\{a, b, c\}$, we have $b P_{2}^{1} d$.

If $n$ is odd, then suppose $\left|S^{\prime}\right|=(n / 2)-2$ and let $P_{2}^{1} \in \mathcal{D}_{2}$ be such that:

$c P_{2}^{1} a P_{2}^{1} b$ and for each $d \in A \backslash\{a, b, c\}$, we have $b P_{2}^{1} d$.

Let $z$ of $F$ be a function satisfying conditions (i) to (iii) of Definition 4. Also, let $C \in \mathcal{C}$ be such that $C_{1}=\left(P_{1}^{1}, P_{1}^{2}, \ldots\right)$ and for each $i \in N \backslash\{1\}$, we have $C_{i}=\left(P_{i}^{1}, \ldots\right)$. By assumption, $s_{2}>0$. Thus, $F\left(\left(P_{i}^{1}\right)_{i \in N}\right)=\{a, b\}$ and $F\left(P_{1}^{2},\left(P_{i}^{1}\right)_{i \in N \backslash\{1\}}\right)=\{a\}$. By conditions $(i)$ and $(i i), z(b,(1, \ldots, 1), C)=b$ and $z(b,(2,1, \ldots, 1), C)=a$. Thus, $z(b,(1, \ldots, 1), C) P_{1}^{2}$ $z(b,(2,1, \ldots, 1), C)$, contradicting condition (iv) of Definition 4 , hence $F$ violates strong chain dominance.

This completes the proof.

Note that Ohseto (1994) proves that the plurality correspondence is not implementable with respect to material preferences if $n>2$ and $|A|>2$. First, he proves that this SCC 
fails to satisfy strategy-resistance if $(n=4$ and $|A|>2),(n \geq 6$ and $|A|>2)$, or $(n=5$ and $|A|>3$ ). Second, he proves by contradiction that this SCC cannot be implemented if $(n=3$ and $|A|>2)$ or $(n=5$ and $|A|=3)$. This corresponds to Cases 3 and 4 of the proof of Theorem 3.5 in Ohseto (1994) respectively. One can prove this by using strong chain dominance. Indeed, one can prove that in Case 3 of the proof of this theorem, the plurality correspondence violates this condition using the same logic as in Case 1 above. This is however not true for a positional scoring rule with $s_{1}>s_{2}>0$. Case 2 above corresponds to the example in Case 4 of the proof of this theorem.

\section{References}

Bok, S. (1999). Lying: Moral Choice in Public and Private Life. Vintage.

Börgers, T. (1991). Undominated strategies and coordination in normalform games. Social Choice and Welfare, 8:65-78.

Börgers, T. and Smith, D. (2012). Robustly ranking mechanisms. American Economic Review: Papers and Proceedings, 102:325-329.

Carroll, G. (2014). A complexity result for undominated-strategy implementation. Mimeo.

Doghmi, A. and Ziad, A. (2013). On partially honest Nash implementation in private good economies with restricted domains: A sufficient condition. The B.E. Journal of Theoretical Economics, 13:415-428.

Dutta, B. and Sen, A. (2012). Nash implementation with partially honest individuals. Games and Economic Behavior, 74:154-169.

Gibbard, A. (1973). Manipulation of voting schemes: A general result. Econometrica, 41:587-602.

Gneezy, U. (2005). Deception: The role of consequences. The American Economic Review, $95: 384-394$.

Hurkens, S. and Kartik, N. (2009). Would I lie to you? On social preferences and lying aversion. Experimental Economics, 12:180-192.

Jackson, M. O. (1992). Implementation in undominated strategies: A look at bounded mechanisms. The Review of Economic Studies, 59:757-775. 
Kartik, N., Tercieux, O., and Holden, R. (2014). Simple mechanisms and preferences for honesty. Games and Economic Behavior, 83:284-290.

Koray, S. and Slinko, A. (2008). Self-selective social choice functions. Social Choice and Welfare, 31:129-149.

Korpela, V. (2014). Bayesian implementation with partially honest individuals. Social Choice and Welfare, 43:647-658.

Levine, T. R., Kim, R. K., and Hamel, L. M. (2010). People lie for a reason: Three experiments documenting the principle of veracity. Communication Research Reports, $27: 271-285$.

Lombardi, M. and Yoshihara, N. (2014). Partially honest Nash implementation: A full characterization. Mimeo.

Lombardi, M. and Yoshihara, N. (2016). Treading a fine line: (im)possibilities for Nash implementation with partially-honest individuals. Mimeo.

Lombardi, M. and Yoshihara, N. (2017). Natural implementation with semi-responsible agents in pure exchange economies. International Journal of Game Theory. Forthcoming.

Matsushima, H. (2008a). Behavioral aspects of implementation theory. Economics Letters, 100:161-164.

Matsushima, H. (2008b). Role of honesty in full implementation. Journal of Economic Theory, 139:353-359.

McCornack, S. A. (1997). The generation of deceptive messages: Laying the groundwork for a viable theory of interpersonal deception. In Greene, J. O., editor, Message production: Advances in communication theory, pages 91-126. Lawrence Erlbaum Associates, Mahwah, NJ.

Ohseto, S. (1994). Implementation of the plurality correspondence in undominated strategies by a bounded mechanism. The Economic Studies Quarterly, 45:97-105.

Ortner, J. (2015). Direct implementation with minimally honest individuals. Games and Economic Behavior, 90:1-16. 
Satterthwaite, M. (1975). Strategy-proofness and Arrow's conditions: Existence and correspondence theorems for voting procedures and social welfare functions. Journal of Economic Theory, 10:187-217.

Thomson, W. (1996). Concepts of implementation. The Japanese Economic Review, 47:133143.

Yamashita, T. (2012). A necessary condition for implementation in undominated strategies, with applications to robustly optimal trading mechanisms. Unpublished manuscript.

Yamashita, T. (2015). Implementation in weakly undominated strategies: Optimality of second-price auction and posted-price mechanism. The Review of Economic Studies, 82:1223-1246. 